\title{
Kinetic temperature of massive star-forming molecular clumps measured with formaldehyde
}

\section{The Orion molecular cloud $1^{\star}$}

\author{
X. D. Tang ${ }^{1,2,3}$, C. Henkel ${ }^{1,4}$, K. M. Menten ${ }^{1}$, F. Wyrowski ${ }^{1}$, N. Brinkmann ${ }^{1}$, X. W. Zheng ${ }^{5}$, Y. Gong ${ }^{1,6}$, Y. X. Lin ${ }^{1}$, \\ J. Esimbek ${ }^{2,3}$, J. J. Zhou ${ }^{2,3}$, Y. Yuan ${ }^{2,3}$, D. L. Li ${ }^{2,3}$, and Y. X. He ${ }^{2,3}$ \\ 1 Max-Planck-Institut für Radioastronomie, Auf dem Hügel 69, 53121 Bonn, Germany \\ e-mail: xdtang@mpifr-bonn.mpg.de \\ 2 Xinjiang Astronomical Observatory, Chinese Academy of Sciences, 830011 Urumqi, PR China \\ 3 Key Laboratory of Radio Astronomy, Chinese Academy of Sciences, 830011 Urumqi, PR China \\ 4 Astronomy Department, King Abdulaziz University, PO Box 80203, 21589 Jeddah, Saudi Arabia \\ 5 Department of Astronomy, Nanjing University, 210093 Nanjing, PR China \\ ${ }^{6}$ Purple Mountain Observatory \& Key Laboratory for Radio Astronomy, Chinese Academy of Sciences, 210008 Nanjing, PR China
}

Received 28 August 2017 / Accepted 22 September 2017

\begin{abstract}
We mapped the kinetic temperature structure of the Orion molecular cloud 1 (OMC-1) with para- $\mathrm{H}_{2} \mathrm{CO}\left(J_{K_{\mathrm{a}} K_{\mathrm{c}}}=3_{03}-2_{02}, 3_{22}-2_{21}\right.$, and $\left.3_{21}-2_{20}\right)$ using the APEX $12 \mathrm{~m}$ telescope. This is compared with the temperatures derived from the ratio of the $\mathrm{NH}_{3}(2,2) /(1,1)$ inversion lines and the dust emission. Using the RADEX non-LTE model, we derive the gas kinetic temperature modeling the measured averaged line ratios of para- $\mathrm{H}_{2} \mathrm{CO}_{22}-2_{21} / 3_{03}-2_{02}$ and $3_{21}-2_{20} / 3_{03}-2_{02}$. The gas kinetic temperatures derived from the para- $\mathrm{H}_{2} \mathrm{CO}$ line ratios are warm, ranging from 30 to $>200 \mathrm{~K}$ with an average of $62 \pm 2 \mathrm{~K}$ at a spatial density of $10^{5} \mathrm{~cm}^{-3}$. These temperatures are higher than those obtained from $\mathrm{NH}_{3}(2,2) /(1,1)$ and $\mathrm{CH}_{3} \mathrm{CCH}(6-5)$ in the OMC-1 region. The gas kinetic temperatures derived from para- $\mathrm{H}_{2} \mathrm{CO}$ agree with those obtained from warm dust components measured in the mid infrared (MIR), which indicates that the para- $\mathrm{H}_{2} \mathrm{CO}(3-2)$ ratios trace dense and warm gas. The cold dust components measured in the far infrared (FIR) are consistent with those measured with $\mathrm{NH}_{3}(2,2) /(1,1)$ and the $\mathrm{CH}_{3} \mathrm{CCH}(6-5)$ line series. With dust at MIR wavelengths and para- $\mathrm{H}_{2} \mathrm{CO}(3-2)$ on one side, and dust at FIR wavelengths, $\mathrm{NH}_{3}(2,2) /(1,1)$, and $\mathrm{CH}_{3} \mathrm{CCH}(6-5)$ on the other, dust and gas temperatures appear to be equivalent in the dense gas $\left(n\left(\mathrm{H}_{2}\right) \gtrsim 10^{4} \mathrm{~cm}^{-3}\right)$ of the OMC-1 region, but provide a bimodal distribution, one more directly related to star formation than the other. The non-thermal velocity dispersions of para- $\mathrm{H}_{2} \mathrm{CO}$ are positively correlated with the gas kinetic temperatures in regions of strong non-thermal motion (Mach number $\gtrsim 2.5$ ) of the OMC-1, implying that the higher temperature traced by para- $\mathrm{H}_{2} \mathrm{CO}$ is related to turbulence on a $\sim 0.06 \mathrm{pc}$ scale. Combining the temperature measurements with para- $\mathrm{H}_{2} \mathrm{CO}$ and $\mathrm{NH}_{3}(2,2) /(1,1)$ line ratios, we find direct evidence for the dense gas along the northern part of the OMC-1 $10 \mathrm{~km} \mathrm{~s}^{-1}$ filament heated by radiation from the central Orion nebula.
\end{abstract}

Key words. stars: formation - stars: massive - ISM: clouds - ISM: molecules - radio lines: ISM

\section{Introduction}

At a distance of $\approx 400 \mathrm{pc}$ (Menten et al. 2007; Kounkel et al. 2017), Orion A and B are the nearest giant molecular clouds (GMCs; e.g., O'Dell et al. 2008). Within Orion A, Orion molecular cloud 1 (OMC-1) abuts the Orion nebula (M42), a prominent $\mathrm{H}$ iI region that harbors the Trapezium O-/early B-type stars and is ionized by one of them, $\theta_{1} \mathrm{C}$ Ori. This star also excites the Orion Bar, a dense photon-dominated region (PDR), which is a protrusion of OMC-1 in which the neutral cloud acquires a nearly edge-on geometry, so that the optically thin PDR emission is limb-brightened. Star formation in OMC-1 is thought to be taking place in two locations: in the Kleinmann-Low (KL) Nebula and in Orion South. While evidence exists for lowor intermediate-mass star formation in Orion-S (Zapata et al. 2004), the amount of star formation activity in KL is under debate. In particular, Zapata et al. (2011) have challenged the

\footnotetext{
* The reduced datacubes are only available at the CDS via anonymous ftp to cdsarc.u-strasbg. fr (130.79.128.5) or via http://cdsarc.u-strasbg.fr/viz-bin/qcat?]/A+A/609/A16
}

view that the famous "hot core" located in that region is powered by a young high-mass stellar object.

OMC-1 is a unique region for studying the physical and chemical conditions of molecular clouds. A large number of molecular line observations have been performed, such as in CO (e.g., Bally et al. 1987; Goldsmith et al. 1997; Wilson et al. 2005; Peng et al. 2012; Buckle et al. 2012; Shimajiri et al. 2011，2014; Berné et al. 2014), CS (e.g., Tatematsu et al. 1993, 1998), $\mathrm{N}_{2} \mathrm{H}^{+}$(e.g., Tatematsu et al. 2008; Melnick et al. 2011; Hacar et al. 2017), $\mathrm{NH}_{3}$ (e.g., Batrla et al. 1983; Bastien et al. 1985; Murata et al. 1990; Wiseman \& Ho 1996, 1998; Batrla \& Wilson 2003; Friesen et al. 2017), $\mathrm{H}_{2} \mathrm{CO}$ (e.g., Thaddeus et al. 1971; Kutner et al. 1976; Cohen \& Few 1981; Batrla et al. 1983; Bastien et al. 1985; Mangum et al. 1990, 1993; van der Wiel et al. 2009; Leurini et al. 2010), $\mathrm{HC}_{3} \mathrm{~N}$ (e.g., Martín-Pintado et al. 1990; Rodríguez et al. 1992; Bergin et al. 1996), and HCN (e.g., Schilke et al. 1992; Melnick et al. 2011). These observations revealed the distribution of the dense gas within OMC-1 down to a $\sim 0.1 \mathrm{pc}$ scale. In addition to molecular line observations, (sub)millimeter 
continuum observations have revealed remarkable filamentary structure over the entire Orion A molecular cloud with a length of $\sim 7$ pc (e.g., Lis et al. 1998; Johnston \& Bally 1999; Shimajiri et al. 2011; Salji et al. 2015; Stutz \& Kainulainen 2015; Kainulainen et al. 2017).

Ammonia $\left(\mathrm{NH}_{3}\right)$ is frequently used as the standard molecular cloud thermometer (e.g., Ho \& Townes 1983; Walmsley \& Ungerechts 1983; Danby et al. 1988; Mangum et al. 2013b). However, it has shown a large spread in fractional abundance (e.g., $10^{-5}$ in dense molecular "hot cores" around newly formed massive stars, Mauersberger et al. $1987 ; 10^{-8}$ in dark clouds, Benson \& Myers 1983; Chira et al. 2013; $10^{-10}$ in the Orion Bar PDR, Batrla \& Wilson 2003) and is extremely affected by a high UV flux. In contrast, formaldehyde $\left(\mathrm{H}_{2} \mathrm{CO}\right)$ is a more ubiquitous molecule in the interstellar medium (Downes et al. 1980; Bieging et al. 1982; Cohen et al. 1983; Baan et al. 1986, 1990, 1993; Henkel et al. 1991; Zylka et al. 1992; Mangum et al. 2008, 2013a; Zhang et al. 2012; Ao et al 2013; Tang et al. 2013; Ginsburg et al. 2015, 2016; Guo et al. 2016). Unlike ammonia, the fractional abundance of $\mathrm{H}_{2} \mathrm{CO}$ is stable at various stages of star formation (Mangum et al. 1990, 1993; Caselli et al. 1993; Johnstone et al. 2003; Gerner et al. 2014; Tang et al. 2017a,b, 2018). OMC-1 has been a valuable target for measuring lines of $\mathrm{H}_{2} \mathrm{CO}$ because of its high densities and temperatures (e.g., Mangum et al. 1990, 1993; Bergin et al. 1994, 1996; Peng et al. 2012; Gong et al. 2015b; Kauffmann et al. 2017) and subsequently large surface brightnesses (e.g., Lis et al. 1998; Johnston \& Bally 1999; Megeath et al. 2012; Lombardi et al. 2014). Previous observations show that $\mathrm{H}_{2} \mathrm{CO}$ has a spatially extensive distribution in the OMC-1 region including Orion KL, Orion South, the Orion Bar, and the northern part of the OMC-1 region (e.g., Batrla et al. 1983; Bastien et al. 1985; Mangum et al. 1990, 1993; van der Wiel et al. 2009; Leurini et al. 2010).

Formaldehyde is a reliable probe for tracing physical conditions of molecular clouds (Henkel et al. 1980, 1983; Mangum \& Wootten 1993; Mühle et al. 2007; Ginsburg et al. 2011). It has a rich variety of millimeter and submillimeter transitions. Particularly useful are the three transitions of para$\mathrm{H}_{2} \mathrm{CO}\left(J_{K_{2} K_{\mathrm{c}}}=3_{03}-2_{02}, 3_{22}-2_{21}\right.$, and $\left.3_{21}-2_{20}\right)$, which can be measured simultaneously at $\sim 218 \mathrm{GHz}$ with a bandwidth of $1 \mathrm{GHz}$. These transitions of para- $\mathrm{H}_{2} \mathrm{CO}$ may trace denser regions than $\mathrm{NH}_{3}(1,1) /(2,2)$ (e.g., Ott et al. 2014; Ginsburg et al. 2016). Since the relative populations of the $K_{\mathrm{a}}$ ladders of $\mathrm{H}_{2} \mathrm{CO}$ are governed by collisions, line ratios involving different $K_{\mathrm{a}}$ ladders are good tracers of the kinetic temperature (Mangum \& Wootten 1993; Mühle et al. 2007). Therefore the line strength ratios of para- $\mathrm{H}_{2} \mathrm{CO}, 3_{22}-2_{21} / 3_{03}-2_{02}$ and $3_{21}-2_{20} / 3_{03}-2_{02}$, provide a sensitive thermometer, possibly the best of the very few which are available for the analysis of dense molecular gas. These $\mathrm{H}_{2} \mathrm{CO}$ line ratios have been used to measure physical parameters in our Galactic center clouds (Qin et al. 2008; Ao et al. 2013; Johnston et al. 2014; Ginsburg et al. 2016; Immer et al. 2016; Lu et al. 2017), star formation regions (Mangum \& Wootten 1993; Hurt et al. 1996; Mitchell et al. 2001; Watanabe \& Mitchell 2008; Lindberg et al 2015; Tang et al. 2017a, 2018), as well as in external galaxies (Mühle et al. 2007; Tang et al. 2017b).

Many observations have been performed to reveal the temperatures of gas and dust in the Orion region (e.g., Downes et al. 1981; Churchwell \& Hollis 1983; Bergin et al. 1994; Wiseman \& Ho 1998; Mookerjea et al. 2000; Vaillancourt 2002; Batrla \& Wilson 2003; Megeath et al. 2012; Peng et al. 2012; Lombardi et al. 2014; Goicoechea et al. 2015; Nishimura et al.
2015; Salgado et al. 2016; Friesen et al. 2017). However, critical links between $\mathrm{H}_{2} \mathrm{CO}$ and other measurements of gas temperatures as well as dust temperatures are still unclear. In this paper, we aim to map the kinetic temperature structure of the OMC-1 region with three transitions of para- $\mathrm{H}_{2} \mathrm{CO}\left(J_{K_{\mathrm{a}} K_{\mathrm{c}}}=3_{03}-2_{02}, 3_{22}-\right.$ $2_{21}$, and $\left.3_{21}-2_{20}\right)$. In Sects. 2 and 3 , we introduce our observation of the para- $\mathrm{H}_{2} \mathrm{CO}$ triplet, data reduction, and results. We discuss the resulting kinetic temperatures derived from para- $\mathrm{H}_{2} \mathrm{CO}$ in Sect. 4. Our main conclusions are summarized in Sect. 5. This paper is part of the "Kinetic temperature of massive star-forming molecular clumps measured with formaldehyde" series of studies exploring $\mathrm{H}_{2} \mathrm{CO}$ as a probe of gas conditions in a variety of sources.

\section{Observations and data reduction}

Our observations were carried out in 2015 October with the APEX $^{1} 12 \mathrm{~m}$ telescope located on Chajnantor (Chile) using the APEX-1 (SHeFI) receiver. The beam size is $\sim 28.5^{\prime \prime}(\sim 0.06 \mathrm{pc}$ at $\sim 400$ pc distance; see Menten et al. 2007; Kounkel et al. 2017). The main beam efficiency and the forward efficiency were 0.75 and 0.97 , respectively. The para- $\mathrm{H}_{2} \mathrm{CO} J_{K_{\mathrm{a}} K_{\mathrm{c}}}=3_{03}-2_{02}, 3_{22}-$ 221 , and $3_{21}-2_{20}$ transitions have rest frequencies of 218.222 , 218.475 , and $218.760 \mathrm{GHz}$, respectively, which are measured simultaneously by employing the eXtended bandwidth Fast Fourier Transform Spectrometer (XFFTS) backend for two spectral windows of $2.5 \mathrm{GHz}$ bandwidth. The frequency range, consisting of two spectral windows, covered $216.5-219.0 \mathrm{GHz}$ and $218.0-220.5 \mathrm{GHz}$ with the central frequency being set to $218.550 \mathrm{GHz} .32768$ spectral channels were used in each window providing a velocity resolution of $\sim 0.1 \mathrm{~km} \mathrm{~s}^{-1}$. The on-thefly observing mode was used to measure eight $4.5 \times 3.5 \mathrm{arcmin}^{2}$ maps with steps of $9^{\prime \prime}$ in both right ascension and declination. The surveyed area of the OMC-1 is $\sim 9 \times 14 \operatorname{arcmin}^{2}(\sim 1.1 \times$ $\left.1.7 \mathrm{pc}^{2}\right)$, centered on $\alpha_{2000}=05: 35: 12.50, \delta_{2000}=-05: 22: 55.0$. The observed spectrum toward Orion KL is shown in Fig. 1.

Data reduction for spectral lines and maps was performed with GILDAS ${ }^{2}$. The spectra were resampled in steps of $\sim 14.2^{\prime \prime}$. To enhance signal to noise ratios $(\mathrm{S} / \mathrm{Ns})$ in individual channels, we smoothed contiguous channels to a velocity resolution $\sim 0.4 \mathrm{~km} \mathrm{~s}^{-1}$. A typical rms noise level $(1 \sigma)$ is $\sim 0.13 \mathrm{~K}$ ( $T_{\mathrm{mb}}$ scale) for a channel of $0.4 \mathrm{~km} \mathrm{~s}^{-1}$ in width. In total we acquired 2472 positions, corresponding to 2472 spectra for each transition. We fit all spectra with Gaussian profiles. Nearly $50 \%$ of all positions were detected in para- $\mathrm{H}_{2} \mathrm{CO} 3_{03}-2_{02}$ and $\sim 16 \%$ were also detected in para- $\mathrm{H}_{2} \mathrm{CO} 3_{22}-2_{21}$ and $3_{21}-2_{20}$, respectively, with $\mathrm{S} / \mathrm{Ns}$ of $\gtrsim 3 \sigma$.

\section{Results}

\subsection{Distribution of $\mathrm{H}_{2} \mathrm{CO}$}

The intensity distributions of the three transitions of para$\mathrm{H}_{2} \mathrm{CO}$ integrated from 4 to $14 \mathrm{~km} \mathrm{~s}^{-1}$ in the OMC-1 are shown in Fig. 2. The para- $\mathrm{H}_{2} \mathrm{CO}_{30}-2_{02}$ line shows an extended distribution and clearly traces the dense molecular structure, for example, Orion KL, Orion South, the Orion Bar,

\footnotetext{
This publication is based on data acquired with the Atacama Pathfinder EXperiment (APEX). APEX is a collaboration between the Max-Planck-Institut für Radioastronomie, the European Southern Observatory, and the Onsala Space Observatory.

2 http://www.iram. fr/IRAMFR/GILDAS
} 


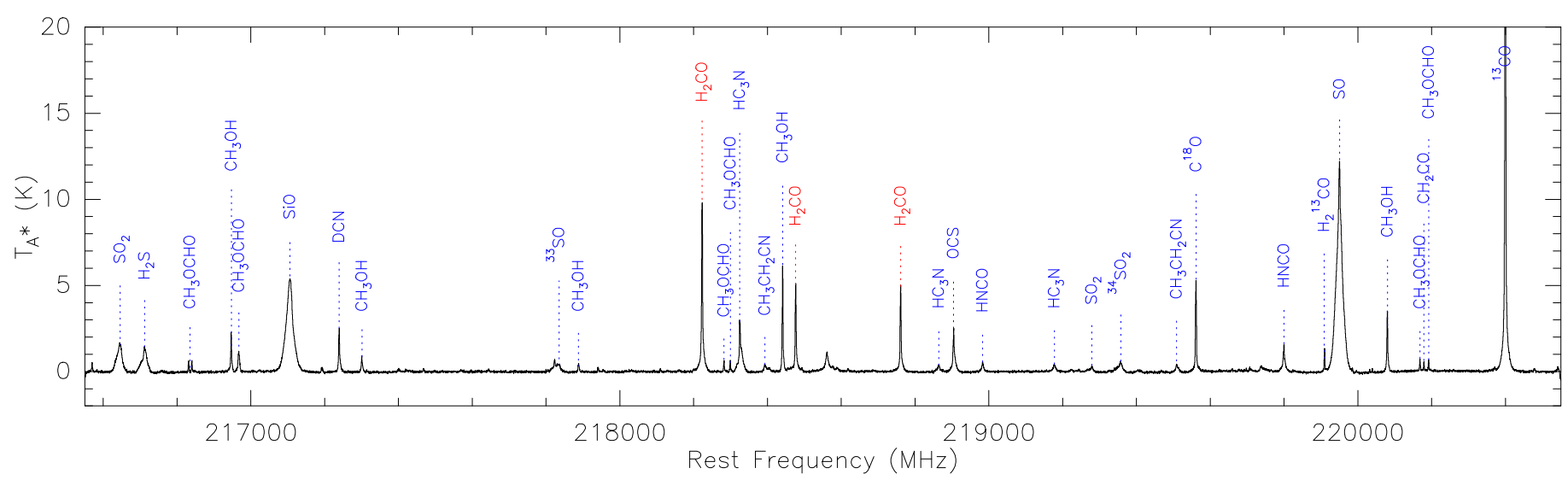

Fig. 1. A spectrum toward Orion KL obtained with APEX (Atacama Pathfinder EXperiment). The three $\mathrm{H}_{2} \mathrm{CO}$ lines discussed in this paper are marked in red.

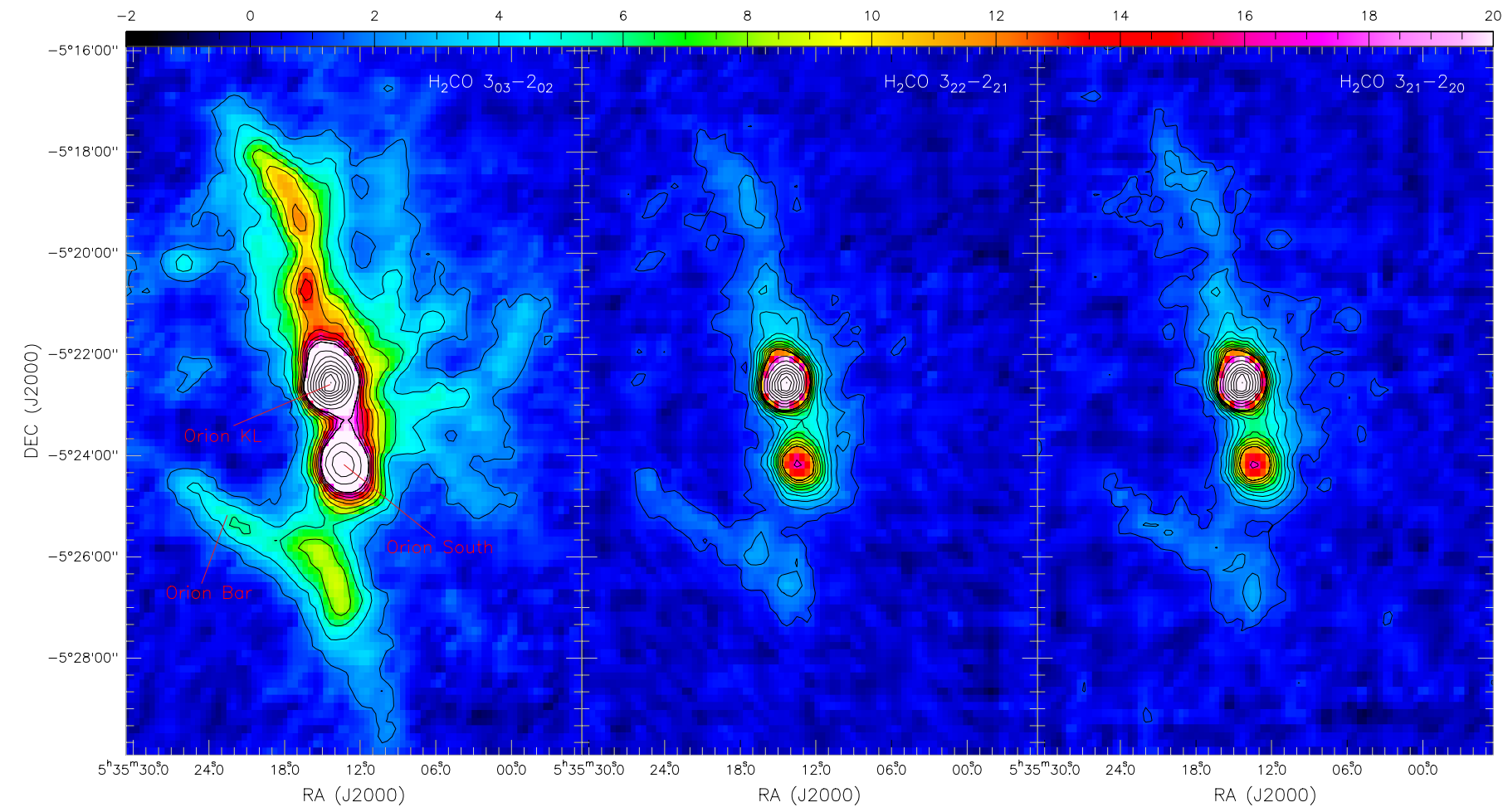

Fig. 2. Intensity maps ( $T_{\mathrm{A}}^{*}$ scale; color bar in units of $\mathrm{K} \mathrm{km} \mathrm{s}^{-1}$ ) of para- $\mathrm{H}_{2} \mathrm{CO} 3_{03}-2_{02}$ (left), $3_{22}-2_{21}$ (middle), and $3_{21}-2_{20}$ (right) integrated from $V_{\mathrm{LSR}}=4$ to $14 \mathrm{~km} \mathrm{~s}^{-1}$ of the OMC-1 cloud. Contour levels are from 1.9 to $19 \mathrm{~K} \mathrm{~km} \mathrm{~s}^{-1}$ with steps of $1.9 \mathrm{~K} \mathrm{~km} \mathrm{~s}^{-1}$ and from 19 to $95 \mathrm{~K} \mathrm{~km} \mathrm{~s}^{-1}$ with steps of $9.5 \mathrm{~K} \mathrm{~km} \mathrm{~s}^{-1}$ for para- $\mathrm{H}_{2} \mathrm{CO}_{303}-2_{02}$, and from 1.05 to $10.5 \mathrm{~K} \mathrm{~km} \mathrm{~s}^{-1}$ with steps of $1.05 \mathrm{~K} \mathrm{~km} \mathrm{~s}^{-1}$ and from 10.5 to $52.5 \mathrm{~K} \mathrm{~km} \mathrm{~s}^{-1}$ with steps of $5.25 \mathrm{~K} \mathrm{~km} \mathrm{~s}^{-1}$ for para- $\mathrm{H}_{2} \mathrm{CO} 3_{22}-2_{21}$ and $3_{21}-2_{20}$.

and molecular fingers, which confirms previous observational results of the dense gas distribution traced by $\mathrm{H}_{2} \mathrm{CO}$ (e.g., Thaddeus et al. 1971; Kutner et al. 1976; Batrla et al. 1983; Bastien et al. 1985; Mangum et al. 1990; van der Wiel et al. 2009; Leurini et al. 2010). It is also consistent with previous observational results probed with other dense gas tracers of, for example, $\mathrm{C}^{18} \mathrm{O}, \mathrm{CS}, \mathrm{CN}, \mathrm{HCN}, \mathrm{HNC}, \mathrm{HCO}^{+}, \mathrm{HC}_{3} \mathrm{~N}$, and $\mathrm{CH}_{3} \mathrm{CCH}$ (Martín-Pintado et al. 1990; Rodríguez et al. 1992; Schilke et al. 1992; Tatematsu et al. 1993, 1998; Bergin et al. 1994, 1996; Goldsmith et al. 1997; Ungerechts et al. 1997; Buckle et al. 2012; Peng et al. 2012; Shimajiri et al. 2014). Para$\mathrm{H}_{2} \mathrm{CO} 3_{22}-2_{21}$ and $3_{21}-2_{20}$ are only detected in the densest regions of the OMC-1, and show less extended distributions than para- $\mathrm{H}_{2} \mathrm{CO} 3_{03}-2_{02}$.
The para- $\mathrm{H}_{2} \mathrm{CO} 3_{03}-2_{02}$ intensity-weighted mean velocity (moment 1 ), and velocity dispersion (moment 2 ) maps are shown in Fig. 3, revealing complex structure. Figure A.1 shows the corresponding channel maps. The northeastern region and the star forming regions Orion KL and Orion South with their outflows are prominent at low velocities $\left(\sim 6-9 \mathrm{~km} \mathrm{~s}^{-1}\right)$, while the Orion Bar and the northern region become visible at higher velocities $\left(\sim 9-11 \mathrm{~km} \mathrm{~s}^{-1}\right)$. The velocity dispersion of para- $\mathrm{H}_{2} \mathrm{CO}\left(3_{03}-2_{02}\right)$ ranges from 0.7 to $6 \mathrm{~km} \mathrm{~s}^{-1}$ with an average of $\sim 2 \mathrm{~km} \mathrm{~s}^{-1}$. Only the star forming regions Orion KL and Orion South are prominent at high velocity dispersions $\left(>3 \mathrm{~km} \mathrm{~s}^{-1}\right)$. In other dense regions of the OMC-1, the velocity dispersions become $\sim 1.5-$ $3 \mathrm{~km} \mathrm{~s}^{-1}$. The outer parts of the OMC-1 show a lower range of velocity dispersion $\left(<1.5 \mathrm{~km} \mathrm{~s}^{-1}\right)$. 

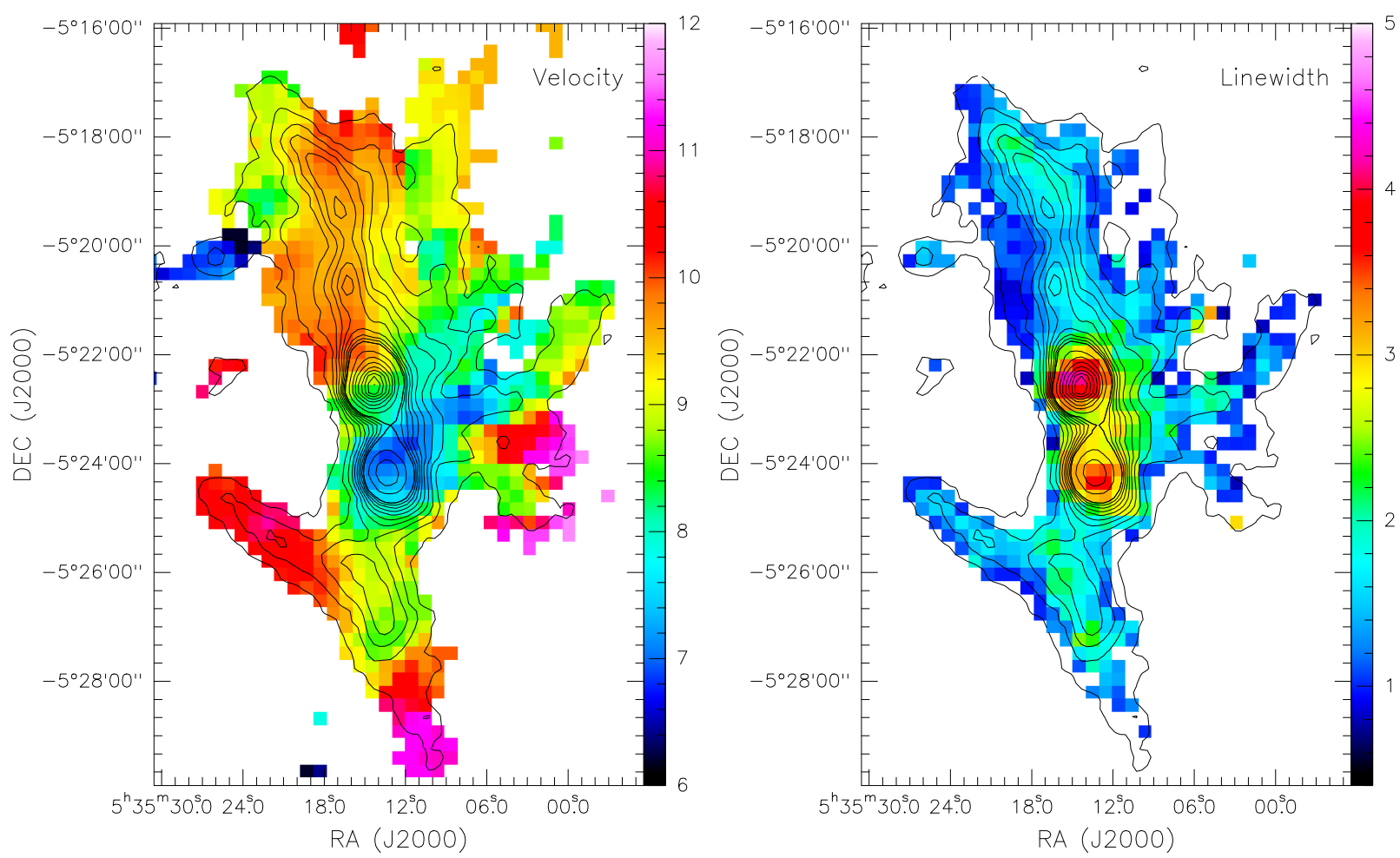

Fig. 3. Para- $\mathrm{H}_{2} \mathrm{CO} 3_{03}-2_{02}$ intensity-weighted mean velocity map (moment 1 , left) and velocity dispersion map (moment 2 , right). The unit of each color bar is $\mathrm{km} \mathrm{s}^{-1}$. Contours are para- $\mathrm{H}_{2} \mathrm{CO}_{303}-2_{02}$ integrated intensities (same as in Fig. 2).

The distribution of para- $\mathrm{H}_{2} \mathrm{CO}\left(3_{03}-2_{02}\right)$ is similar to the spatial distribution of the $\mathrm{NH}_{3}(1,1)$ and $(2,2)$ emission mapped with the Green Bank Telescope (GBT; beam size 30"; Friesen et al. 2017) (see Fig. B.1) and all prominent features identified in the $\mathrm{NH}_{3}(1,1)$ and $(2,2)$ lines are detected in para$\mathrm{H}_{2} \mathrm{CO}\left(3_{03}-2_{02}\right)$ at a scale of $\sim 0.06 \mathrm{pc}$ in the OMC- 1 . The similar distributions of $\mathrm{H}_{2} \mathrm{CO}\left(3_{03}-2_{02}\right)$ and $\mathrm{NH}_{3}(1,1)$ and $(2,2)$ in the OMC-1 region are consistent with previous observational results in the Galactic Central Molecular Zone (CMZ) on a $\sim 0.1 \mathrm{pc}$ scale ( $\mathrm{Lu}$ et al. 2017). The strongest $\mathrm{H}_{2} \mathrm{CO}\left(3_{03}-2_{02}\right)$ emission associates with the massive star formation regions Orion $\mathrm{KL}$ and Orion South, which is consistent with the $\mathrm{NH}_{3}(2,2)$ emission. However, the strongest $\mathrm{NH}_{3}(1,1)$ emission is found toward Orion $\mathrm{KL}$ and the northern clumps, which is slightly different from what we see in $\mathrm{H}_{2} \mathrm{CO}\left(3_{03}-2_{02}\right)$. The agreement between the distributions of para- $\mathrm{H}_{2} \mathrm{CO}\left(3_{03}-2_{02}\right)$ and $\mathrm{NH}_{3}(2,2)$ is better than that between para- $\mathrm{H}_{2} \mathrm{CO}\left(3_{03}-2_{02}\right)$ and $\mathrm{NH}_{3}(1,1)$ (see Fig. B.1). The probable reason is that $\mathrm{NH}_{3}(1,1)$ may sample a more extended and lower-density gas.

The para- $\mathrm{H}_{2} \mathrm{CO}\left(3_{03}-2_{02}\right)$ integrated intensity distribution agrees remarkably well with the 450 and $850 \mu \mathrm{m}$ dust emission (Johnston \& Bally 1999) in the OMC-1 region, including the dense molecular structure and dust emission peaks (see Fig. B.1). This confirms previous observational results in massive star-forming clumps at various evolutionary stages (Tang et al. 2017a, 2018).

\subsection{Kinetic temperature}

As discussed in Sect. 1, the relative intensity ratio of $\mathrm{H}_{2} \mathrm{CO}$ lines involving different $K_{\mathrm{a}}$ ladders yields estimates of the kinetic temperature of the gas (Mangum \& Wootten 1993). The para- $\mathrm{H}_{2} \mathrm{CO}_{22}-2_{21}$ and $3_{21}-2_{20}$ transitions have similar upper state energies above the ground state, $E_{\mathrm{u}} \simeq 68 \mathrm{~K}$, similar spatial distributions (see Fig. 2), similar line profiles (brightness temperature, linewidth, and velocity in our observations; see Fig. C.1; also see Tang et al. 2017a,b, 2018), and are often detected together in molecular clouds (e.g., Bergman et al. 2011; Wang et al. 2012; Lindberg \& Jørgensen 2012; Ao et al. 2013; Immer et al. 2014; Treviño-Morales et al. 2014; Ginsburg et al. 2016, 2017; Tang et al. 2017a,b, 2018; Lu et al. 2017). The $\mathrm{CH}_{3} \mathrm{OH} 4_{22}-3_{12}$ transition at $218.440 \mathrm{GHz}$ is well separated from the para- $\mathrm{H}_{2} \mathrm{CO}\left(3_{22}-2_{21}\right)$ transition in the OMC-1 region (see Figs. 1 and C.1). Para- $\mathrm{H}_{2} \mathrm{CO}_{22}-2_{21} / 3_{03}-2_{02}$ and $3_{21}-2_{20} / 3_{03}-2_{02}$ ratios are good thermometers to determine kinetic temperature and show a similar behavior to kinetic temperature and spatial density at high density $n\left(\mathrm{H}_{2}\right) \gtrsim 10^{5} \mathrm{~cm}^{-3}$ (Lindberg et al. 2015; Tang et al. 2017a), so in this work we use the averaged ratio $0.5 \times\left[\left(3_{22}-2_{21}+3_{21}-2_{20}\right) / 3_{03}-2_{02}\right]$ between para- $\mathrm{H}_{2} \mathrm{CO} 3_{22}-2_{21} / 3_{03}-2_{02}$ and $3_{21}-2_{20} / 3_{03}-2_{02}$ to determine gas kinetic temperatures.

Using the RADEX ${ }^{3}$ non-local thermodynamical equilibrium (LTE) model (van der Tak et al. 2007) with collision rates from Wiesenfeld \& Faure (2013), we modeled the relation between the gas kinetic temperature and the measured average of para- $\mathrm{H}_{2} \mathrm{CO} 0.5 \times\left[\left(3_{22}-2_{21}+3_{21}-2_{20}\right) / 3_{03}-2_{02}\right]$ ratios, adopting an average measured linewidth of $\sim 2.0 \mathrm{~km} \mathrm{~s}^{-1}$ (temperature weakly dependent on the linewidth; see Nagy et al. 2012; Immer et al. 2016) and column densities $N\left(\right.$ para- $\left.\mathrm{H}_{2} \mathrm{CO}\right)=1 \times$ $10^{13}, 5 \times 10^{13}$, and $1 \times 10^{14} \mathrm{~cm}^{-2}$ in Fig. 4. Previous observations toward the Galactic CMZ clouds and dense massive starforming clumps show that the opacities of para- $\mathrm{H}_{2} \mathrm{CO}(3-2)$ lines weakly influence the measurements of gas kinetic temperature (Ginsburg et al. 2016; Immer et al. 2016; Tang et al. 2018), so here we assume that the para- $\mathrm{H}_{2} \mathrm{CO}(3-2)$ lines are optically

3 http://var.sron.nl/radex/radex.php 


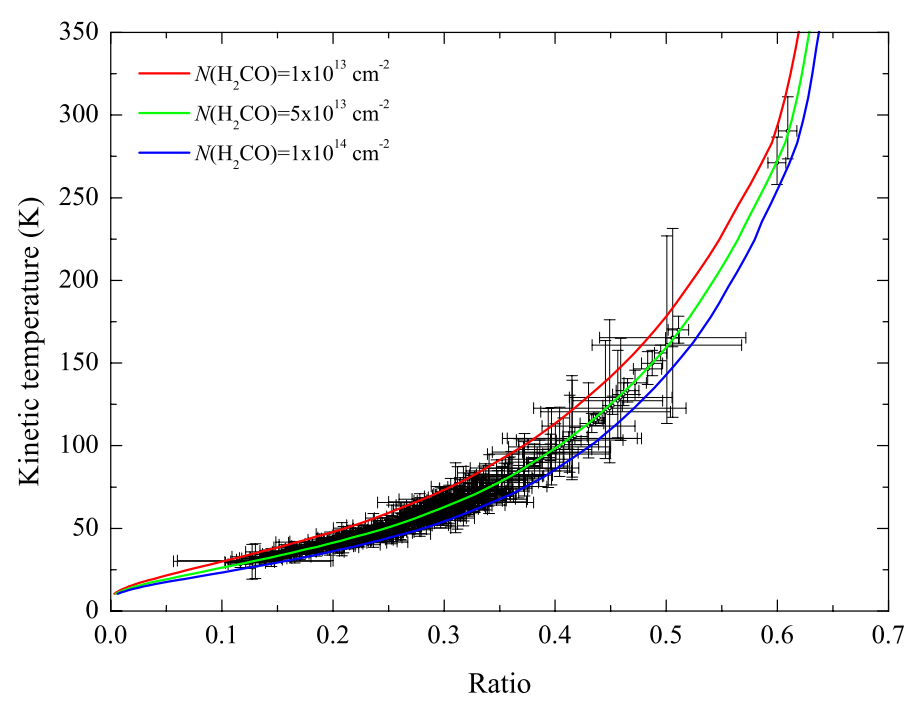

Fig. 4. RADEX non-LTE modeling of the relation between the kinetic temperature and the average ratio of para- $\mathrm{H}_{2} \mathrm{CO}_{22}-2_{21} / 3_{03}-$ $2_{02}$ and $3_{21}-2_{20} / 3_{03}-2_{02}$ with an assumed density of $n\left(\mathrm{H}_{2}\right)=10^{5} \mathrm{~cm}^{-3}$, an averaged linewidth of $2.0 \mathrm{~km} \mathrm{~s}^{-1}$, and column density of $N$ (para$\left.\mathrm{H}_{2} \mathrm{CO}\right)=1 \times 10^{13}$ (red line), $5 \times 10^{13}$ (green line), and $1 \times 10^{14} \mathrm{~cm}^{-2}$ (blue line). The black points are derived from our observed $\mathrm{H}_{2} \mathrm{CO}$ line ratios for a column density $N\left(\right.$ para- $\left.\mathrm{H}_{2} \mathrm{CO}\right)=5 \times 10^{13} \mathrm{~cm}^{-2}$, the average value for the OMC-1 (see Sect. 3.2). The temperature uncertainties are obtained from observed para- $\mathrm{H}_{2} \mathrm{CO}$ line ratio errors.

thin in the OMC-1 region. The spatial densities measured with $\mathrm{H}_{2} \mathrm{CO}$ and $\mathrm{HC}_{3} \mathrm{~N}$ in the OMC-1 region are $n\left(\mathrm{H}_{2}\right) \sim 10^{5}-$ $10^{6} \mathrm{~cm}^{-3}$ (Mangum et al. 1993; Bergin et al. 1996). Previous observations show that para- $\mathrm{H}_{2} \mathrm{CO}(3-2)$ is sensitive to gas at a density of $10^{5} \mathrm{~cm}^{-3}$ (Ginsburg et al. 2016; Immer et al. 2016; Tang et al. 2017b). Therefore, we adopt $10^{5} \mathrm{~cm}^{-3}$ as an average gas spatial density in the OMC-1 region. The average column density $N\left(\right.$ para- $\left.\mathrm{H}_{2} \mathrm{CO}\right)$ obtained from the para- $\mathrm{H}_{2} \mathrm{CO}\left(3_{03}-2_{02}\right)-$ averaged brightness temperatures of the entire OMC- 1 region at a density of $10^{5} \mathrm{~cm}^{-3}$ using the method of Tang et al. (2017a) is $\sim 5 \times 10^{13} \mathrm{~cm}^{-2}$. The temperatures derived from different column densities at a density of $10^{5} \mathrm{~cm}^{-3}$ would only slightly change (see Fig. 4). Generally, higher line ratios of para- $\mathrm{H}_{2} \mathrm{CO}$ indicate higher kinetic temperatures. Therefore, the ratio maps can be used as a proxy for relative kinetic temperature. We use the relation between kinetic temperature and para- $\mathrm{H}_{2} \mathrm{CO}$ line ratios at a spatial density of $10^{5} \mathrm{~cm}^{-3}$ and column density of $5 \times 10^{13} \mathrm{~cm}^{-2}$ (shown in Fig. 5) to convert ratio maps to temperature maps in Fig. 5.

An averaged para- $\mathrm{H}_{2} \mathrm{CO}(3-2)$ line ratio map is shown in Fig. 5. The line ratios are calculated by velocity-integrated intensities where the para- $\mathrm{H}_{2} \mathrm{CO} 3_{22}-2_{21}$ and/or $3_{21}-2_{20}$ lines are detected with $S / N \gtrsim 3 \sigma$. Para- $\mathrm{H}_{2} \mathrm{CO}$ ratios range from 0.12 to 0.61 , with an average of $0.28 \pm 0.01$ (errors given here and elsewhere are standard deviations of the mean). The lowest ratios $(<0.25)$ occur in the Orion north(west) and the ridge between Orion South and the Orion Bar. In Orion South, in the north(east), and in the dense clumps in the Orion Bar, the ratios range from 0.25 to 0.4 . High ratios $(>0.4)$ are also found in the northeastern region, Orion KL, and the Orion Bar. Several locations near the hot core, in the northeastern region, and in the Orion Bar around the $\mathrm{H}$ il region show the highest ratios $(>0.45)$.

The gas kinetic temperatures derived from the para- $\mathrm{H}_{2} \mathrm{CO}$ line ratios are warm, ranging from 30 to $>200 \mathrm{~K}$ with an average of $62 \pm 2 \mathrm{~K}$ at density $n\left(\mathrm{H}_{2}\right)=10^{5} \mathrm{~cm}^{-3}$, which agrees with the results measured with $\mathrm{H}_{2} \mathrm{CO}$ in other star-forming regions
(Mangum \& Wootten 1993; Hurt et al. 1996; Mangum et al. 1999; Watanabe \& Mitchell 2008; Nagy et al. 2012) and Galactic center clouds (Ao et al. 2013; Ginsburg et al. 2016; Immer et al. 2016; Lu et al. 2017). The kinetic temperatures in the dense gas around the $\mathrm{H}$ II region and in the northeastern part of the OMC-1 $10 \mathrm{~km} \mathrm{~s}^{-1}$ filament are high (>50 K; see Fig. 5 and Table 1). Typical kinetic temperatures are $\sim 120-290 \mathrm{~K}$ in Orion $\mathrm{KL}, \sim 74 \mathrm{~K}$ in Orion South, $\sim 73 \mathrm{~K}$ in dense clumps of the Orion Bar, $\sim 100-130 \mathrm{~K}$ at the edge (north and south) of the Orion Bar, $\sim 40 \mathrm{~K}$ in Orion north, and $\sim 70-160 \mathrm{~K}$ in the Orion northeastern region.

\section{Discussion}

\subsection{Comparison of temperatures derived from $\mathrm{H}_{2} \mathrm{CO}$ and other gas tracers}

The $\mathrm{NH}_{3}(2,2) /(1,1)$ ratio is sensitive to gas temperatures $T_{\text {kin }}<50 \mathrm{~K}$ (Mangum et al. 2013a; Gong et al. 2015a), which is similar to the kinetic temperature range that the para$\mathrm{H}_{2} \mathrm{CO}(3-2)$ ratio is most sensitive to (Mangum \& Wootten 1993). The $\mathrm{NH}_{3}$ lines have lower effective excitation densities than the para- $\mathrm{H}_{2} \mathrm{CO}(3-2)$ transitions by a few orders of magnitude, $n_{\mathrm{eff}}\left(\mathrm{NH}_{3}(1,1)\right) \sim 10^{3} \mathrm{~cm}^{-1}$ while $n_{\mathrm{eff}}\left(\right.$ para- $\mathrm{H}_{2} \mathrm{CO} 3_{03}-$ $\left.2_{02}\right) \sim 10^{5} \mathrm{~cm}^{-1}$ (Shirley 2015). The Orion A molecular cloud has been measured in $\mathrm{NH}_{3}(1,1)$ and $(2,2)$ with the GBT (beam size $\sim 30^{\prime \prime}$; Friesen et al. 2017; this agrees well with our para$\mathrm{H}_{2} \mathrm{CO}$ (3-2) data). We compare maps of gas kinetic temperatures derived from para- $\mathrm{H}_{2} \mathrm{CO}$ and $\mathrm{NH}_{3}(2,2) /(1,1)$ line ratios in Fig. 5. The typical gas kinetic temperature derived from $\mathrm{NH}_{3}(2,2) /(1,1)$ is $20-30 \mathrm{~K}$. Typical gas kinetic temperatures are $>100 \mathrm{~K}$ in Orion KL, $\sim 50 \mathrm{~K}$ in Orion South, $>50 \mathrm{~K}$ in the Orion Bar, $20-30 \mathrm{~K}$ in Orion north and $>50 \mathrm{~K}$ in the Orion northeastern region (see Table 1). Almost everywhere, $\mathrm{NH}_{3}(2,2) /(1,1)$ traces lower kinetic temperatures than those derived from para$\mathrm{H}_{2} \mathrm{CO}(3-2)$ line ratios. We also compare matched pixels of kinetic temperature maps of the two tracers in Fig. D.1. It seems that temperatures derived from the two tracers are correlated. In many cases, para- $\mathrm{H}_{2} \mathrm{CO}(3-2)$ traces a higher temperature than $\mathrm{NH}_{3}(2,2) /(1,1)$ with a difference of $5->100 \mathrm{~K}$. Previous observations toward Galactic CMZ clouds, dense massive star-forming clumps in our Galactic disk and external galaxies indicate that in many cases para- $\mathrm{H}_{2} \mathrm{CO}(3-2)$ line ratios trace a higher kinetic temperature than the $\mathrm{NH}_{3}(2,2) /(1,1)$ line ratios (Weiß et al. 2001; Mühle et al. 2007; Ao et al. 2013; Ott et al. 2014; Ginsburg et al. 2016; Tang et al. 2017a,b, 2018). The difference is likely due to the fact that the derived kinetic temperatures from $\mathrm{NH}_{3}(2,2) /(1,1)$ reflect an average temperature of more extended and cooler gas (Henkel et al. 1987; Ginsburg et al. 2016) and para- $\mathrm{H}_{2} \mathrm{CO}(3-2)$ ratios trace denser and hotter regions (Ginsburg et al. 2016; Tang et al. 2017a, 2018).

A comparison of temperatures obtained from $\mathrm{NH}_{3}(4,4) /$ $(2,2)$ and para- $\mathrm{H}_{2} \mathrm{CO}\left(3_{21}-2_{20} / 3_{03}-2_{02}\right)$ line ratios in a Galactic CMZ cloud indicates that in many cases the tracers reveal consistent results (Lu et al. 2017). Kinetic temperatures in the Orion Bar have been measured with the $\mathrm{NH}_{3}(4,4) /(2,2)$ ratio (Batrla \& Wilson 2003), which shows similar temperatures as derived from the para- $\mathrm{H}_{2} \mathrm{CO}(3-2)$ ratio. More highly excited $\mathrm{NH}_{3}$ transitions commonly lead to higher kinetic temperatures (e.g., Henkel et al. 1987; Mangum et al. 2013a; Gong et al. 2015a,b). Therefore, if higher $\mathrm{NH}_{3}$ levels than $\mathrm{NH}_{3}(2,2) /(1,1)$ are involved in measuring the kinetic temperatures, the values derived from $\mathrm{NH}_{3}$ might become at least as high as those from para- $\mathrm{H}_{2} \mathrm{CO}(3-2)$ in the OMC-1 region. 

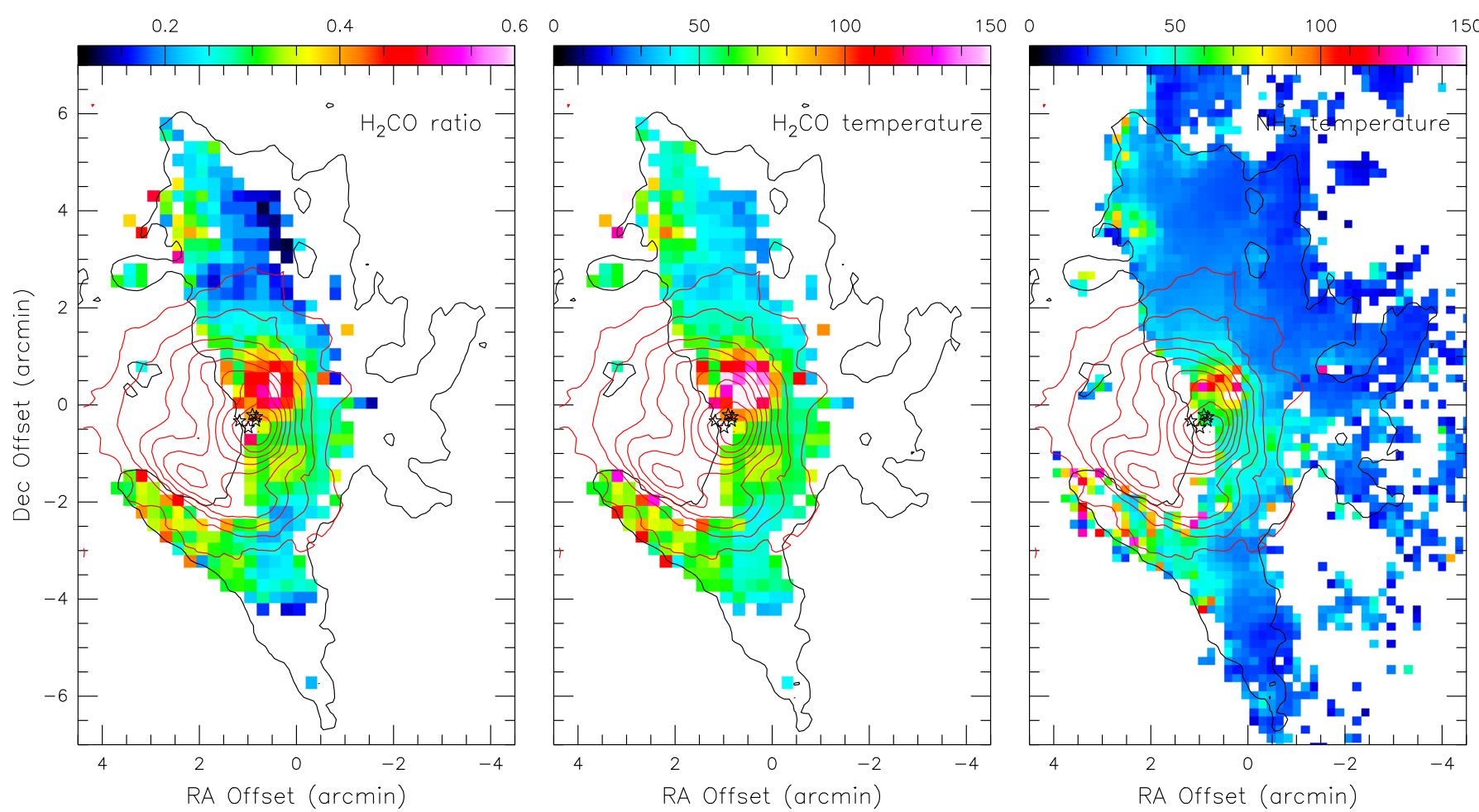

Fig. 5. Left: averaged (see Sect. 3.2) velocity-integrated intensity ratio map of para- $\mathrm{H}_{2} \mathrm{CO} 0.5 \times\left[\left(3_{22}-2_{21}+3_{21}-2_{20}\right) / 3_{03}-2_{02}\right]$ in the $\mathrm{OMC}-1$. Middle: kinetic temperatures derived from the para- $\mathrm{H}_{2} \mathrm{CO}(3-2)$ line ratios. Right: kinetic temperatures derived from $\mathrm{NH}_{3}(2,2) /(1,1)$ ratios observed with the GBT (beam size $\sim 30^{\prime \prime}$; Friesen et al. 2017). Black contours show the contour of integrated intensity of para- $\mathrm{H}_{2} \mathrm{CO} 3_{03}-2_{02}$ at $1.9 \mathrm{~K} \mathrm{~km} \mathrm{~s}^{-1}$ (see Fig. 2). Red contours show the velocity-integrated intensity $\left(V_{\mathrm{LSR}}=-30\right.$ to $\left.+30 \mathrm{~km} \mathrm{~s}^{-1}\right)$ of the $\mathrm{H} 41 \alpha$ recombination line observed with IRAM (beam size 30"; Goicoechea et al. 2015). Stars show the locations of the Trapezium stars ( $\theta^{1}$ Ori A, B, C, D, and E). The unit of the color bars of the middle and right panels is in both cases Kelvin.

Table 1. Comparison of temperatures derived from different tracers.

\begin{tabular}{cccccccc}
\hline \hline Region & $\mathrm{H}_{2} \mathrm{CO}$ & $\mathrm{NH}_{3}$ & $\mathrm{CH}_{3} \mathrm{CCH}$ & $\mathrm{CO}(1-0)$ & $\mathrm{CO}(6-5)$ & $\mathrm{MIR}$ & $\mathrm{FIR}$ \\
& $\mathrm{K}$ & $\mathrm{K}$ & $\mathrm{K}$ & $\mathrm{K}$ & $\mathrm{K}$ & $\mathrm{K}$ & $\mathrm{K}$ \\
\hline $\mathrm{KL}$ & $120-290$ & $>100$ & $50-60$ & $78-88$ & $>150$ & $>150$ & $45-60$ \\
South & $\sim 74$ & $\sim 50$ & $\sim 46$ & $81-92$ & $>150$ & $\sim 75$ & $\sim 30$ \\
North & $\sim 40$ & $20-30$ & $13-40$ & $43-60$ & $\ldots$ & $\ldots$ & $26-30$ \\
Northeast & $70-160$ & $>50$ & $40-51$ & $60-78$ & $\ldots$ & $\ldots$ & $30-44$ \\
Bar (clump) & $\sim 73$ & $>50$ & $\ldots$ & $\ldots$ & $>150$ & $\sim 70$ & $33-50$ \\
Bar (edge) & $100-130$ & $>100$ & $\ldots$ & $\ldots$ & $>100$ & $>100$ & $41-51$ \\
\hline
\end{tabular}

Notes. Column 2: kinetic temperatures derived from para- $\mathrm{H}_{2} \mathrm{CO}$ line ratios (see Sect. 3.2). Column 3: kinetic temperatures derived from the $\mathrm{NH}_{3}(2,2) /(1,1)$ line ratios taken from Friesen et al. (2017). Column 4: rotation temperatures obtained from $\mathrm{CH}_{3} \mathrm{CCH}(6-5)$ taken from Bergin et al. (1994). Columns 5 and 6: excitation temperatures of $C O(1-0)$ and $C O(6-5)$ taken from Bergin et al. (1994) and Peng et al. (2012), respectively. Columns 7 and 8: mid (MIR) and far infrared (FIR) dust temperatures taken from Downes et al. (1981), Vaillancourt (2002), Lombardi et al. (2014), Salgado et al. (2016), and Kauffmann et al. (2017).

Several locations in the Orion $\mathrm{KL}$ region have been observed in $\mathrm{CH}_{3} \mathrm{CCH} \quad(J=5-4$ and 6-5; Churchwell \& Hollis 1983), which is also a thermometer tracing dense molecular gas. It reveals a gas temperature of $\sim 35 \mathrm{~K}$. A region similar to that of our observations has been observed in $\mathrm{CH}_{3} \mathrm{CCH}(6-5$, beam size 50"; Bergin et al. 1994), suggesting a gas temperature range of $10-60 \mathrm{~K}$ with an average of $\sim 30 \pm 1 \mathrm{~K}$, which is similar to results obtained from $\mathrm{NH}_{3}(2,2) /(1,1)$ line ratios but is lower than that derived from our para- $\mathrm{H}_{2} \mathrm{CO}$ line ratios. This difference is also found in dense massive star-forming clumps (Tang et al. 2018; Giannetti et al. 2017). These indicate that, like the $\mathrm{NH}_{3}(2,2) /(1,1)$ ratio, $\mathrm{CH}_{3} \mathrm{CCH}(6-5)$ might trace cooler and more extended gas (Churchwell \& Hollis 1983).

$\mathrm{CO}(1-0)$ with a beam size of $\sim 45^{\prime \prime}$ has been observed toward the OMC-1 region (Bergin et al. 1994), which reveals a warm gas temperature (equivalent to the excitation temperature since ${ }^{12} \mathrm{CO}$ is likely optically thick) ranging from 43 to $92 \mathrm{~K}$ with an average of $\sim 69 \pm 2 \mathrm{~K}$. This is similar to results derived from para- $\mathrm{H}_{2} \mathrm{CO}(3-2)$ line ratios. Low-resolution (beam size $\sim 3^{\prime}$ ) observations of the $\mathrm{OMC}$ with $\mathrm{CO}(2-1)$ show a gas kinetic temperature of $\sim 88 \mathrm{~K}$ at a density of $\sim 10^{3} \mathrm{~cm}^{-3}$ in the Orion KL region (Nishimura et al. 2015), which agrees with our averaged gas temperature $(\sim 82 \mathrm{~K})$ derived from the para- $\mathrm{H}_{2} \mathrm{CO}(3-$ 2) line ratio at a density of $10^{5} \mathrm{~cm}^{-3}$ within a similar region. Observations of the high excitation $\mathrm{CO}(6-5)$ transition (beam size $\left.\sim 8.6^{\prime \prime}\right)$ toward the OMC-1 region indicate extensive high gas temperatures (excitation temperature $>150 \mathrm{~K}$ ) all over Orion KL, Orion South, and the Orion Bar regions (Peng et al. 2012), whereas in $\mathrm{H}_{2} \mathrm{CO}$ (and also $\mathrm{NH}_{3}$ ) we only see such high $T_{\text {kin }}$ values in the Orion KL region. This suggests that para- $\mathrm{H}_{2} \mathrm{CO}(3-2)$ 
X. D. Tang et al.: Kinetic temperature in OMC-1
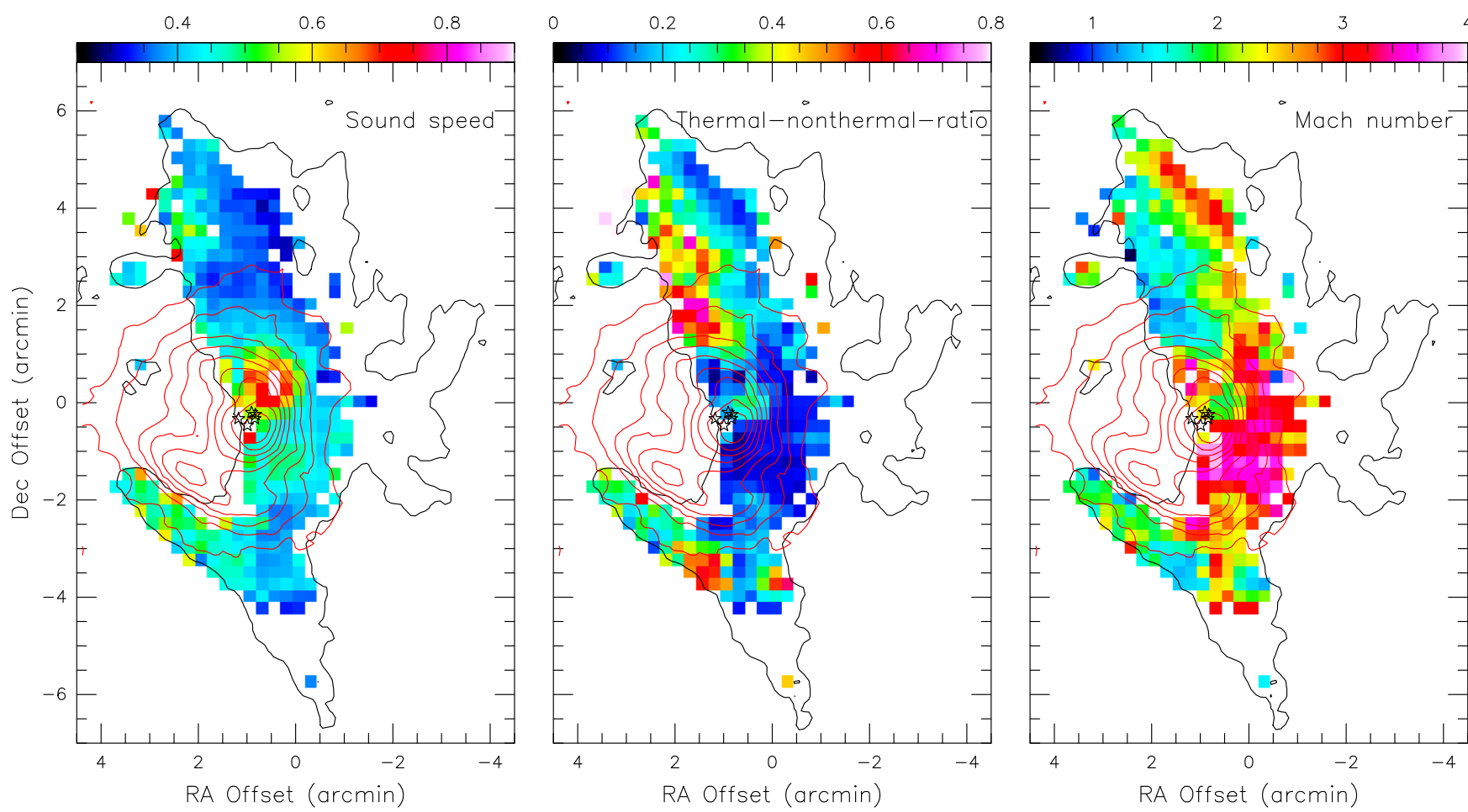

Fig. 6. Maps of sound speed (left; color bar in units of $\mathrm{km} \mathrm{s}^{-1}$ ), thermal to non-thermal gas pressure (middle), and Mach number (right) in the OMC-1. Contours and stars are the same as in Fig. 5. For details, see Sect. 4.3.

may trace a cooler component than the high-excitation transition of $\mathrm{CO}(6-5)$.

\subsection{Comparison of temperatures derived from the gas and the dust}

The dust temperature in the Orion A molecular cloud has been well studied from far-infrared (FIR) to mid-infrared (MIR) wavelengths (e.g., Downes et al. 1981; Mookerjea et al. 2000; Vaillancourt 2002; Megeath et al. 2012; Lombardi et al. 2014; Goicoechea et al. 2015; Salgado et al. 2016). The dust temperatures derived from FIR measurements rarely exceed $50 \mathrm{~K}$ in star formation regions of our Galaxy and external galaxies (e.g., Henkel et al. 1986; Mangum et al. 2013a; Guzmán et al. 2015; Merello et al. 2015; He et al. 2016; König et al. 2017; Lin et al. 2016; Yu \& Xu 2016; Tang et al. 2017a). Likely, the dust emission at FIR wavelengths originates primarily from cold dust components that may not be directly associated with star formation activity (Schnee et al. 2009; Bendo et al. 2012; Mangum et al. 2013a). The cold dust temperatures derived from the FIR measurements have a range of $\sim 14$ $60 \mathrm{~K}$ with a roughly constant value, $\sim 30 \mathrm{~K}$, in the extended parts of OMC-1 (Mookerjea et al. 2000; Vaillancourt 2002; Lombardi et al. 2014; Goicoechea et al. 2015; Salgado et al. 2016), which agrees remarkably well with results obtained from $\mathrm{NH}_{3}(2,2) /(1,1)$ and $\mathrm{CH}_{3} \mathrm{CCH}(6-5)$, but is lower than that derived from para- $\mathrm{H}_{2} \mathrm{CO}(3-2)$ line ratios. This indicates that the gas temperatures derived from $\mathrm{NH}_{3}(2,2) /(1,1)$ and $\mathrm{CH}_{3} \mathrm{CCH}(6-5)$ tend to be related to the cold dust component responsible for FIR emission. The dust emission at mid infrared wavelengths traces primarily warm dust components that may be excited by young stars and clusters (Helou 1986). The warm dust temperatures derived from the MIR measurements range from $\sim 45$ to $\gtrsim 150 \mathrm{~K}$ in the OMC-1 region (Downes et al. 1981; Salgado et al. 2016), which is consistent with results obtained from our para- $\mathrm{H}_{2} \mathrm{CO}$ line ratios. This further confirms that the para- $\mathrm{H}_{2} \mathrm{CO}(3-2)$ ratios trace denser and warmer gas.

At the densest regions $\left(n\left(\mathrm{H}_{2}\right) \gtrsim 10^{4} \mathrm{~cm}^{-3}\right)$ interactions between dust and gas become sufficiently frequent, so it is commonly expected that gas and dust are thermally coupled (Goldsmith 2001). Previous observations show that the temperatures derived from gas and dust are often in agreement in the active dense clumps of Galactic disk clouds (Dunham et al. 2010; Giannetti et al. 2013; Battersby et al. 2014). Based on measurements of dust at MIR and FIR wavelengths and gas detected in the para- $\mathrm{H}_{2} \mathrm{CO}(3-2), \mathrm{NH}_{3}(2,2) /(1,1)$, and $\mathrm{CH}_{3} \mathrm{CCH}(6-5)$ transitions, dust and gas temperatures appear to be generally equivalent in the dense gas $\left(n\left(\mathrm{H}_{2}\right) \gtrsim 10^{4} \mathrm{~cm}^{-3}\right)$ of the OMC-1 region, but yield different values depending on how closely they are related to massive star formation.

\subsection{Thermal and non-thermal motions}

Using the kinetic temperatures derived from the para- $\mathrm{H}_{2} \mathrm{CO}$ line ratios, the thermal and non-thermal linewidth $\left(\sigma_{\mathrm{T}}=\sqrt{\frac{k T_{\mathrm{kin}}}{m_{\mathrm{H}_{2} \mathrm{CO}}}}\right.$ and $\sigma_{\mathrm{NT}}=\sqrt{\frac{\Delta v^{2}}{8 \ln 2}-\sigma_{\mathrm{T}}^{2}} \approx \Delta v / 2.355$, where $k$ is the Boltzmann constant, $T_{\text {kin }}$ is the kinetic temperature of the gas, $m_{\mathrm{H}_{2} \mathrm{CO}}$ is the mass of the formaldehyde molecule, and $\Delta v$ is the measured FWHM linewidth of para- $\mathrm{H}_{2} \mathrm{CO} 3_{03}-2_{02}$ from a Gaussian fit; Pan \& Padoan 2009; Dewangan et al. 2016) ranges are $0.09-0.27 \mathrm{~km} \mathrm{~s}^{-1}$ with an average of $0.12 \pm 0.01 \mathrm{~km} \mathrm{~s}^{-1}$ and $0.34-2.78 \mathrm{~km} \mathrm{~s}^{-1}$ with an average of $0.98 \pm 0.02 \mathrm{~km} \mathrm{~s}^{-1}$, respectively. The thermal linewidth is significantly smaller than the non-thermal linewidth, which indicates that the dense gas traced by para- $\mathrm{H}_{2} \mathrm{CO}$ is dominated by non-thermal motions in the OMC-1 region.

Distributions of the sound speed $\left(a_{\mathrm{S}}=\sqrt{\frac{k T_{\text {kin }}}{\mu m_{\mathrm{H}}}}\right.$, where $\mu=2.37$ is the mean molecular weight for molecular clouds and $m_{\mathrm{H}}$ is the 
mass of the hydrogen atom; Dewangan et al. 2016), the thermal to non-thermal pressure ratio $\left(R_{\mathrm{p}}=a_{\mathrm{s}}^{2} / \sigma_{\mathrm{NT}}^{2}\right.$; Lada et al. 2003), and the Mach number (given as $M=\sigma_{\mathrm{NT}} / a_{\mathrm{s}}$ ) in the OMC-1 are shown in Fig. 6. The sound speed ranges from 0.30 to $0.97 \mathrm{~km} \mathrm{~s}^{-1}$ with an average of $0.44 \pm 0.01 \mathrm{~km} \mathrm{~s}^{-1}$. The thermal to non-thermal pressure ratio ranges from 0.05 to 2.12 with an average of $0.27 \pm 0.01$. The Mach number ranges from 0.7 to 4.3 with an average of $2.3 \pm 0.1$. All this suggests that non-thermal pressure and supersonic non-thermal motions (e.g., turbulence, outflows, shocks, and/or magnetic fields) are dominant in the dense gas traced by para- $\mathrm{H}_{2} \mathrm{CO}$ in the OMC-1 region. A few locations have high thermal to non-thermal pressure ratios $\left(R_{\mathrm{p}} \gtrsim 1\right)$ corresponding to lower Mach numbers $(M \lesssim 1)$, which are located in the eastern edge of the northern part of the OMC-1, in the $10 \mathrm{~km} \mathrm{~s}^{-1}$ filament (see Fig. 6).

The thermal to non-thermal pressure ratio and Mach number show apparent gradients along the northern part of the OMC-1 $10 \mathrm{~km} \mathrm{~s}^{-1}$ filament and the Orion Bar regions (see Fig. 6). This indicates that dense gas probed by para- $\mathrm{H}_{2} \mathrm{CO}$ may be affected by the $\mathrm{H}$ II region (see Figs. 5 and 6). Positions with higher thermal to non-thermal pressure ratio $\left(R_{\mathrm{p}}>0.15\right)$ and lower Mach number $(M<2.5)$ located in the south of the OMC-1 $10 \mathrm{~km} \mathrm{~s}^{-1}$ filament and the Orion Bar region have a higher temperature range ( $>60 \mathrm{~K}$, see Fig. 5) and a lower linewidth range $\left(<2 \mathrm{~km} \mathrm{~s}^{-1}\right.$, see Fig. 3). This gas may be heated by FUV photons originating from the $\mathrm{H}_{\text {II }}$ region. Low thermal to non-thermal pressure ratios $\left(R_{\mathrm{p}}<0.15\right)$ and higher Mach numbers $(M>2.5)$ are associated with the outflows of the massive star forming regions Orion South and Orion KL, and the northwestern part of the OMC-1 $10 \mathrm{~km} \mathrm{~s}^{-1}$ filament. This indicates that dense gas traced by para$\mathrm{H}_{2} \mathrm{CO}$ is strongly influenced by non-thermal motions (e.g., outflows and shocks) in these regions.

\subsection{Turbulent heating}

Correlations between the kinetic temperature and linewidth are expected in the case of conversion of turbulent energy into heat (Güsten et al. 1985; Molinari et al. 1996; Ao et al. 2013; Ginsburg et al. 2016; Immer et al. 2016). Present observations toward Galactic $\mathrm{CMZ}$ clouds with para- $\mathrm{H}_{2} \mathrm{CO}(3-2)$ show that the warm dense gas is heated most likely by turbulence on a scale of $\sim 1 \mathrm{pc}$ (Ao et al. 2013; Ginsburg et al. 2016; Immer et al. 2016). However, high-resolution observations with para- $\mathrm{H}_{2} \mathrm{CO}\left(3_{21}-2_{20} / 3_{03}-2_{02}\right)$ and $\mathrm{NH}_{3}(4,4) /(2,2)$ show no apparent correlation between temperatures and linewidths in a Galactic CMZ cloud at a smaller scale of $\sim 0.1 \mathrm{pc}$ (Lu et al. 2017). Previous observations of, for example, $\mathrm{NH}_{3}, \mathrm{H}_{2} \mathrm{CO}$, and $\mathrm{CH}_{3} \mathrm{CCH}$ in star formation regions suggest that the linewidth is correlated with kinetic temperature (Wouterloot et al. 1988; Molinari et al. 1996; Jijina et al. 1999; Wu et al. 2006; Urquhart et al. 2011, 2015; Wienen et al. 2012; Lu et al. 2014; Tang et al. 2017a, 2018; Giannetti et al. 2017).

We examine whether there is a relationship between turbulence and temperature on a $\sim 0.06 \mathrm{pc}$ scale in the OMC- 1 region. We adopt the non-thermal velocity dispersion $\left(\sigma_{\mathrm{NT}}\right)$ of para$\mathrm{H}_{2} \mathrm{CO}$ in good approximation as a proxy for the turbulence, and the kinetic temperature derived from the para- $\mathrm{H}_{2} \mathrm{CO}$ line ratio as the gas kinetic temperature. As mentioned in Sect. 4.3, the dense gas in locations with lower Mach number $(M<2.5)$ may be influenced by external sources, so here we select positions with strong non-thermal motions $(M \gtrsim 2.5$ corresponding to a thermal to non-thermal pressure ratio $\left.R_{\mathrm{p}} \lesssim 0.15\right)$, which are located near massive star formation regions (Orion KL outflows and Orion
South) and the northern region of the $10 \mathrm{~km} \mathrm{~s}^{-1}$ filament of the OMC-1 ridge (see Fig. 6). The relation between kinetic temperature and non-thermal velocity dispersion is shown in Fig. 7 (blue points; blue fitted lines). For the non-thermal velocity dispersion of para- $\mathrm{H}_{2} \mathrm{CO}$ and kinetic temperature, linear least squares fit results are listed in Table 2. These show that the non-thermal velocity dispersion of para- $\mathrm{H}_{2} \mathrm{CO}$ is significantly positively correlated with the gas kinetic temperatures, especially in the two massive star formation regions Orion KL and Orion South, by a power-law of the form $T_{\mathrm{kin}} \propto \sigma_{\mathrm{NT}}^{0.76-1.26}$, which is consistent with results in massive star-forming clumps $\left(T_{\text {kin }} \propto \sigma_{\mathrm{NT}}^{0.67-1.06}\right.$, gas kinetic temperature measured with para- $\mathrm{H}_{2} \mathrm{CO} 3-2$ and 4-3 line ratios, Tang et al. 2018). This implies that the higher temperature traced by para- $\mathrm{H}_{2} \mathrm{CO}$ in the strong non-thermal motion regions of the OMC- 1 is related to the turbulence at a scale of $\sim 0.06 \mathrm{pc}$, which clearly disagrees with at least one cloud from the Galactic center region on a $\sim 0.1 \mathrm{pc}$ scale (see Lu et al. 2017).

We determine the gas kinetic temperature heated by turbulent energy following the method applied by Ao et al. (2013) in their Eq. (20),

$$
\begin{aligned}
T_{\text {turb }}= & \left(\frac{\left(16 \times 10^{-8} a^{3}+7920 a^{0.5} b c^{3} d^{-1}\right)^{1 / 2}}{12 b}\right. \\
& \left.-\frac{4 \times 10^{-4} a^{1.5}}{12 b}\right)^{2 / 3} \mathrm{~K}
\end{aligned}
$$

where the gas density $a$ is in units of $\mathrm{cm}^{-3}$, the velocity gradient $b$ is in units of $\mathrm{km} \mathrm{s}^{-1} \mathrm{pc}^{-1}$, the one-dimensional non-thermal velocity dispersion $c$ is in units of $\mathrm{km} \mathrm{s}^{-1}$, and the cloud size $d$ is in units of pc. This leads to a minimum $T_{\text {turb }}$ value, because the dust temperature has been set to $T_{\text {dust }}=0 \mathrm{~K}$ to simplify the equation (Ao et al. 2013). We computed the gas kinetic temperature assuming turbulent heating dominates the heating process. We adopt a velocity gradient of $b=1 \mathrm{~km} \mathrm{~s}^{-1} \mathrm{pc}^{-1}$ (Bally et al. 1987; Wiseman \& Ho 1998; Hacar et al. 2017) and a gas spatial density of $10^{5} \mathrm{~cm}^{-3}$ (temperature weakly dependent on the velocity gradient and the gas spatial density; see Ao et al. 2013). We select three locations with the largest linewidths of 2.7, 4.4, and $6.6 \mathrm{~km} \mathrm{~s}^{-1}$ at Orion north $\left(1.68^{\prime}, 4.78^{\prime}\right.$ ) (offsets relative to our reference position; see Sect. 2), Orion South $\left(0.72^{\prime},-0.72^{\prime}\right)$, and Orion $\mathrm{KL}\left(0.72^{\prime}, 0.48^{\prime}\right)$, corresponding to gas kinetic temperatures derived from the para- $\mathrm{H}_{2} \mathrm{CO}$ line ratio of 43,62 , and $133 \mathrm{~K}$, respectively. The derived gas kinetic temperature $T_{\text {turb }}$ are 24,58 , and $111 \mathrm{~K}$ at a cloud scale of $0.1 \mathrm{pc}, 9,22$, and $46 \mathrm{~K}$ at a cloud scale of $0.5 \mathrm{pc}$, and 5,14 , and $31 \mathrm{~K}$ at a cloud scale of $1 \mathrm{pc}$ in Orion north, Orion South, and Orion KL, respectively. If the gas temperature derived from para- $\mathrm{H}_{2} \mathrm{CO}$ is adopted as the dust temperature (see Sect. 4.2) following Eqs. (7), (8), (18), and (19) in Ao et al. (2013), Eq. (1) has the form

$$
\begin{aligned}
3.3 \times 10^{-27} a c^{3} d^{-1}= & 4 \times 10^{-33} a^{2} T_{\text {turb }}^{1 / 2}\left(T_{\text {turb }}-T_{\text {dust }}\right) \\
& +6 \times 10^{-29} a^{1 / 2} T_{\text {turb }}^{3} b,
\end{aligned}
$$

and the determined gas kinetic temperatures $T_{\text {turb }}$ become 52 , 87 , and $150 \mathrm{~K}$ at a cloud scale of $0.1 \mathrm{pc}, 42,62$, and $109 \mathrm{~K}$ at a cloud scale of $0.5 \mathrm{pc}$, and 40,57 , and $102 \mathrm{~K}$ at a cloud scale of $1 \mathrm{pc}$ in Orion north, Orion South, and Orion KL, respectively. For the latter two cloud sizes, the obtained $T_{\text {turb }}$ values agree reasonably well with the $T_{\text {kin }}$ values derived from our para- $\mathrm{H}_{2} \mathrm{CO}$ line ratios. This suggests that turbulent heating significantly contributes to the gas kinetic temperature on a small scale. However, these locations are also influenced by, for example, outflows, shocks, and/or radiation from massive star formation regions in 
Table 2. Kinetic temperature vs. $\mathrm{H}_{2} \mathrm{CO}$ non-thermal velocity dispersion.

\begin{tabular}{ccccc}
\hline \hline \multirow{2}{*}{$\begin{array}{c}\mathrm{H}_{2} \mathrm{CO} \\
\text { transition }\end{array}$} & Sample & \multicolumn{3}{c}{$T_{\mathrm{kin}}-\sigma_{\mathrm{NT}}\left(\mathrm{H}_{2} \mathrm{CO}\right)$} \\
\cline { 3 - 5 } & & Slope & Intercept & $R$ \\
\hline $3_{03}-2_{02}$ & OMC-1 & $1.26(0.06)$ & $1.61(0.01)$ & 0.85 \\
& OMC-1+ATLASGAL & $0.89(0.05)$ & $1.64(0.01)$ & 0.81 \\
\hline $3_{22}-2_{21}$ & OMC-1 & $0.76(0.06)$ & $1.69(0.01)$ & 0.72 \\
& OMC-1+ATLASGAL & $0.80(0.05)$ & $1.69(0.01)$ & 0.78 \\
\hline $3_{21}-2_{20}$ & OMC-1 & $0.82(0.07)$ & $1.67(0.01)$ & 0.73 \\
& OMC-1+ATLASGAL & $0.86(0.04)$ & $1.67(0.01)$ & 0.82 \\
\hline
\end{tabular}

Notes. The format of the regression fits is $\log T_{\text {kin }}=$ Slope $\times$ $\log \sigma_{\mathrm{NT}}\left(\mathrm{H}_{2} \mathrm{CO}\right)+$ Intercept. $R$ is the correlation coefficient for the linear fit.

Orion KL and Orion South (Wiseman \& Ho 1998). Nevertheless, turbulent heating may play an important role in heating the dense gas associated with massive star formation regions on small scales in the OMC-1.

To check whether there is a relationship between the turbulence and temperature on scales of 0.06 to $2 \mathrm{pc}$, we combine our data $(M \gtrsim 2.5)$ with previous observational results of para$\mathrm{H}_{2} \mathrm{CO}(3-2)$ in massive star-forming clumps with a Mach number range of 2.7-6.7 at scale $\sim 0.1-1.8 \mathrm{pc}$ (Tang et al. 2018) and fit the relation between the non-thermal velocity dispersion of para- $\mathrm{H}_{2} \mathrm{CO}$ and kinetic temperature in Fig. 7 (red lines). The least squares linear fit results are listed in Table 2. It shows that the non-thermal velocity dispersion of para- $\mathrm{H}_{2} \mathrm{CO}$ is significantly positively correlated with the gas kinetic temperature by a power-law of the form $T_{\mathrm{kin}} \propto \sigma_{\mathrm{NT}}^{0.80-0.89}$ at scale from 0.06 to $1.8 \mathrm{pc}$, which is consistent with our results in the OMC-1 region $(M \gtrsim 2.5)$ and agrees well with results found with para$\mathrm{H}_{2} \mathrm{CO}$ and $\mathrm{NH}_{3}$ in molecular clouds of the Galactic center $\left(T_{\text {kin }} \propto \Delta v^{0.8-1.0}\right.$; Güsten et al. 1985; Mauersberger et al. 1986; Immer et al. 2016). This indicates that turbulent heating seems to be widespread in massive star formation regions on scales of $\sim 0.06-2 \mathrm{pc}$. One should note that this agreement is only in terms of slope, not of intercept and absolute value (see Fig. 7). The fact that the intercept values are different suggests that there are differences in how the gas in the Galactic CMZ clouds and star formation regions of Orion and ATLASGAL sample are heated.

\subsection{Radiation heating}

Previous observations of, for example, $\mathrm{NH}_{3}, \mathrm{H}_{2} \mathrm{CO}, \mathrm{CH}_{3} \mathrm{CN}$, $\mathrm{CH}_{3} \mathrm{CCH}$, or $\mathrm{CH}_{3} \mathrm{OH}$ in massive star-forming regions ( $\mathrm{Lu}$ et al. 2014; Giannetti et al. 2017; Tang et al. 2018) suggest internal radiative heating of embedded infrared sources upon their surrounding dense gas. High-resolution observations with $\mathrm{NH}_{3}(1,1)$ and $(2,2)$ toward the OMC-1 show that the dense gas along the northern part of the OMC-1 $10 \mathrm{~km} \mathrm{~s}^{-1}$ filament is likely heated by radiation from the entire central Orion nebula, including KL and the Trapezium stars (Wiseman \& Ho 1996, 1998).

Following exactly the filament, we investigate the relationship between gas kinetic temperature and distance $R$ from the central part of the Orion nebula (IRc2, $\alpha=05: 35: 14.48$, $\delta=-05: 22: 30.56, \mathrm{~J} 2000)$ along the northern part of the OMC-1 $10 \mathrm{~km} \mathrm{~s}^{-1}$ filament with para- $\mathrm{H}_{2} \mathrm{CO}$ and $\mathrm{NH}_{3}$ in Fig. 8 . The gas kinetic temperatures are derived from para- $\mathrm{H}_{2} \mathrm{CO}$ and $\mathrm{NH}_{3}(2,2) /(1,1)$ (Friesen et al. 2017) line ratios. The beam sizes of para- $\mathrm{H}_{2} \mathrm{CO}$ and $\mathrm{NH}_{3}$ are both $\sim 30^{\prime \prime}$, so we only fit the data for para- $\mathrm{H}_{2} \mathrm{CO}$ and $\mathrm{NH}_{3}$, respectively, with distance $R>30^{\prime \prime}$. For the gas kinetic temperatures of para- $\mathrm{H}_{2} \mathrm{CO}$ and $\mathrm{NH}_{3}$, and
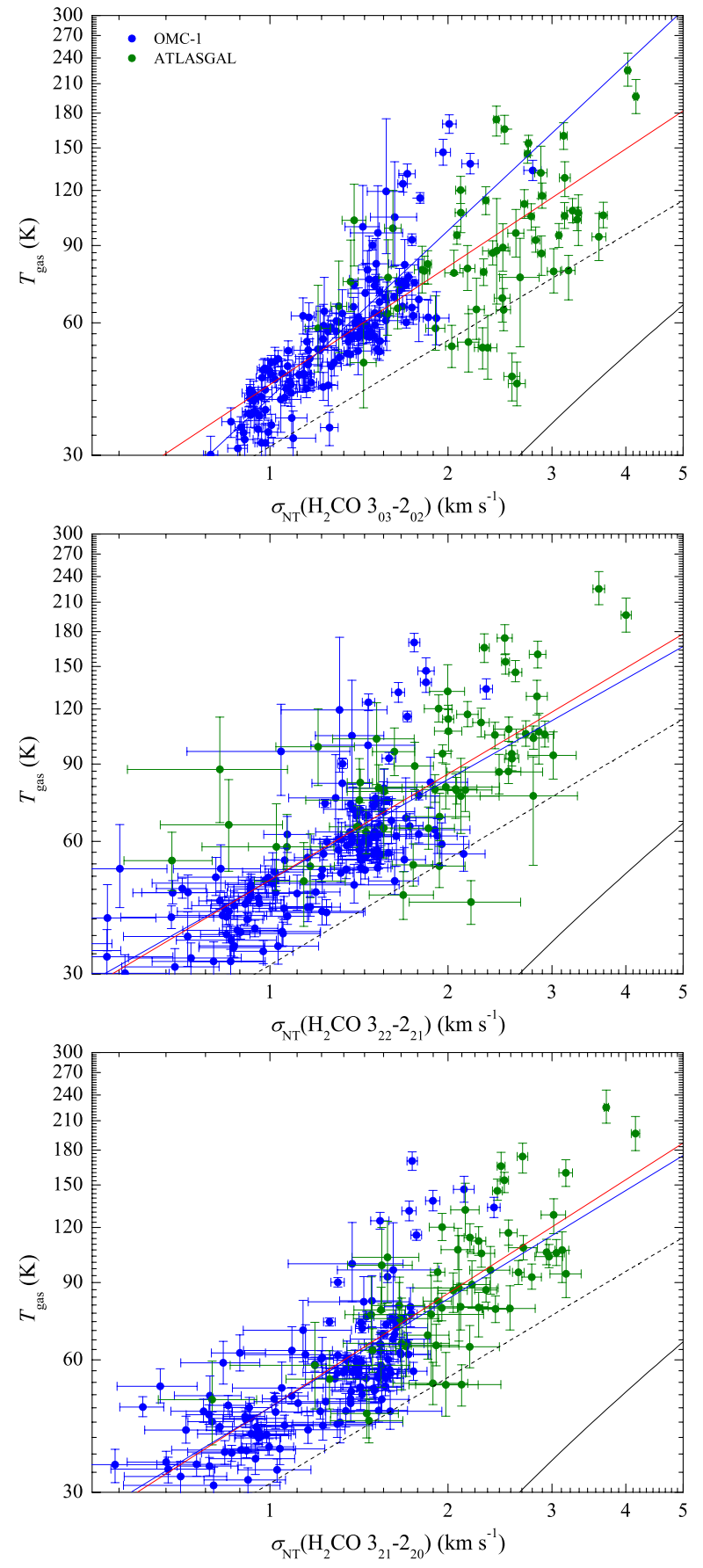

Fig. 7. Non-thermal velocity dispersion $\left(\sigma_{\mathrm{NT}}\right)$ vs. gas kinetic temperature derived from the para- $\mathrm{H}_{2} \mathrm{CO}$ line ratio (Sect. 3.2) for locations with the Mach number $M \gtrsim 2.5$ in the OMC-1 (blue points) and ATLASGAL massive star-forming clumps (green points; Tang et al. 2018). The blue line is the result from a linear fit for the OMC-1 data. The red line is the linearly fitted result for OMC-1 $(M \gtrsim 2.5)$ and ATLASGAL data. Black solid and dashed lines indicate relations between non-thermal velocity dispersion and gas kinetic temperature derived from para- $\mathrm{H}_{2} \mathrm{CO}$ and $\mathrm{NH}_{3}$ at density $10^{5} \mathrm{~cm}^{-3}$ in the Galactic $\mathrm{CMZ}$ clouds, respectively (Güsten et al. 1985; Immer et al. 2016).

distance from IRc2, the fit results are

$T_{\text {kin }}\left(\mathrm{H}_{2} \mathrm{CO}\right)=(57.2 \pm 1.1) \times\left(\frac{R}{\operatorname{arcmin}}\right)^{-0.44 \pm 0.02}$

and

$T_{\text {kin }}\left(\mathrm{NH}_{3}\right)=(45.8 \pm 0.5) \times\left(\frac{R}{\operatorname{arcmin}}\right)^{-0.54 \pm 0.01}$ 


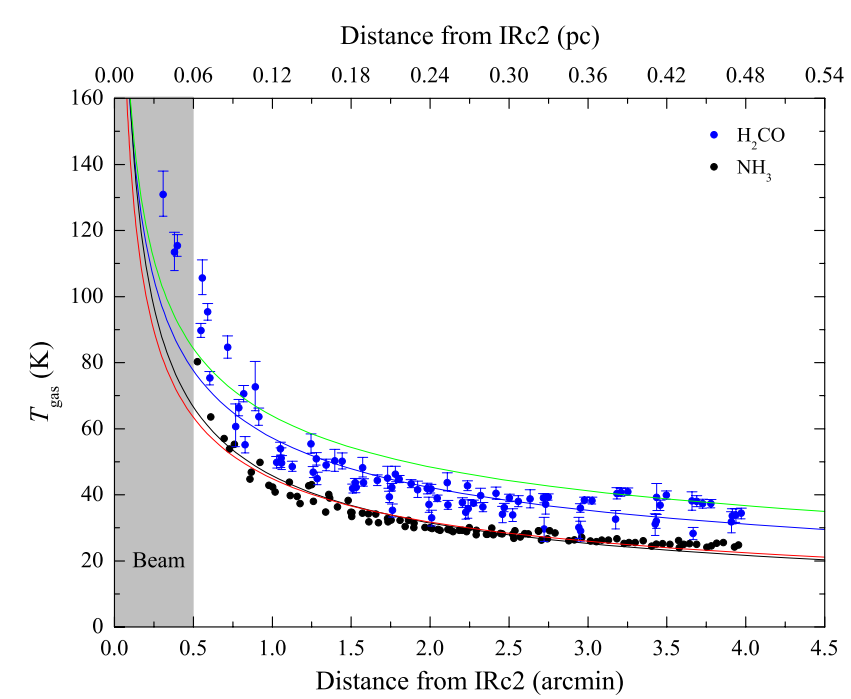

Fig. 8. Gas kinetic temperature derived from para- $\mathrm{H}_{2} \mathrm{CO}(3-2)$ (blue points) and $\mathrm{NH}_{3}(2,2) /(1,1)$ (black points) ratios along the northern $10 \mathrm{~km} \mathrm{~s}^{-1}$ filament of the OMC-1 ridge (distance from IRc2, $\alpha=05: 35: 14.48, \delta=-05: 22: 30.56, \mathrm{~J} 2000)$. The blue and black lines are the fitted results for para- $\mathrm{H}_{2} \mathrm{CO}$ and $\mathrm{NH}_{3}$, respectively, with distance $R>0.5^{\prime}$. The red and green lines are the expected relationships from a Stefan-Boltzmann law and modified Stefan-Boltzmann law (see Sect. 4.5), respectively, assuming Orion KL is the dominant source with an approximate luminosity of $10^{5} L_{\odot}$ (Downes et al. 1981; Wiseman \& Ho 1998).

with power-law indices of -0.44 and -0.54 , respectively. This relation is based on the projected radius along the line of sight.

It is expected that the gas temperature and distance relation from the Stefan-Boltzmann blackbody radiation law is $T_{\text {kin }}=0.86 \times\left(\frac{L}{L_{\odot}}\right)^{1 / 4}\left(\frac{R}{\mathrm{pc}}\right)^{-1 / 2}=44.8 \times\left(\frac{R}{\operatorname{arcmin}}\right)^{-0.5}$, adopting a molecular cloud distance of $400 \mathrm{pc}$ and assuming that IRc2 is the dominant source with an approximate luminosity of $10^{5} L_{\odot}$ (Downes et al. 1981; Wiseman \& Ho 1998), located in the KL nebula. Adjusting the emissivity of dust grains to be smaller than the wavelength at the characteristic black-body temperature, the radiation law has the form $T_{\text {kin }} \approx 63.9 \times\left(\frac{R}{\operatorname{arcmin}}\right)^{-0.4}$ (Wiseman \& Ho 1998). Our fitted power-law indices of para$\mathrm{H}_{2} \mathrm{CO}$ and $\mathrm{NH}_{3}$ are remarkably consistent with the radiation law, which directly confirms that the dense gas along the northern part of the OMC-1 $10 \mathrm{~km} \mathrm{~s}^{-1}$ filament is heated by radiation from the central Orion nebula. The two radiation models for gas heating (Stefan-Boltzmann blackbody radiation and its modification related to dust emissivity) are both well supported by our para$\mathrm{H}_{2} \mathrm{CO}$ and $\mathrm{NH}_{3}$ data, so here we cannot distinguish which is better. For the region with $R \lesssim 1^{\prime}$, several locations show gas temperatures probed with para- $\mathrm{H}_{2} \mathrm{CO}$ above the fitted results (see Figs. 5 and 8). Indeed, the energy set free by an explosion resulting from a stellar merger $\sim 500 \mathrm{yr}$ ago is a plausible mechanism causing the high temperatures of the dense gas in the Orion KL region (Bally \& Zinnecker 2005; Bally et al. 2017).

The gas heating appears to be complex and is most likely due to a number of different processes in the OMC- 1 region. Dense gas around the $\mathrm{H}$ II region at radius $\sim 2.5^{\prime}$, especially the Orion Bar region, appears to be influenced by FUV photons from the Trapezium stars. Star formation activity (e.g., outflows, shocks, winds, radiation) in Orion KL and Orion South also heat the local regions at radius $\gtrsim 2$ ' (Wiseman \& Ho 1998; Bally et al. 2017). Turbulent heating seems to be widespread in the dense gas associated with the massive star formation region in OMC-1.
While dense gas along the northern part of the OMC-1 $10 \mathrm{~km} \mathrm{~s}^{-1}$ filament is heated by radiation from the central Orion nebula.

\section{Summary}

We have mapped the kinetic temperature distribution of OMC-1 with the APEX $12 \mathrm{~m}$ telescope, and compared the kinetic temperatures derived from the para- $\mathrm{H}_{2} \mathrm{CO} 218 \mathrm{GHz}$ line triplet, with $\mathrm{NH}_{3}(2,2) /(1,1)$ inversion lines (Friesen et al. 2017) and dust emission. The main results are the following:

1. The distribution of para- $\mathrm{H}_{2} \mathrm{CO} 3_{03}-2_{02}$ emission is extended in the OMC-1 region and resembles those of the $\mathrm{NH}_{3}(1,1)$ and $(2,2)$ lines on a $\sim 0.06 \mathrm{pc}$ scale.

2. The distribution of para- $\mathrm{H}_{2} \mathrm{CO} 3_{03}-2_{02}$ agrees with the 450 and $850 \mu \mathrm{m}$ dust emission in the OMC-1 region, suggesting that the $\mathrm{H}_{2} \mathrm{CO}$ associates well with dense gas traced by the 450 and $850 \mu \mathrm{m}$ continuum.

3. Using the RADEX non-LTE radiative transfer model, we derive the gas kinetic temperature modeling the measured para- $\mathrm{H}_{2} \mathrm{CO} 0.5 \times\left[\left(3_{22}-2_{21}+3_{21}-2_{20}\right) / 3_{03}-2_{02}\right]$ line ratios. The gas kinetic temperatures derived from para- $\mathrm{H}_{2} \mathrm{CO}$ line ratios are warm, ranging from 30 to $>200 \mathrm{~K}$ with an average of $62 \pm 2 \mathrm{~K}$ at a spatial density of $10^{5} \mathrm{~cm}^{-3}$. These temperatures are higher than those obtained from $\mathrm{NH}_{3}(2,2) /(1,1)$ and $\mathrm{CH}_{3} \mathrm{CCH}(6-5)$ in the OMC-1 region. In many cases para- $\mathrm{H}_{2} \mathrm{CO}(3-2)$ traces a higher temperature than $\mathrm{NH}_{3}(2,2) /(1,1)$ with a difference of $5 \rightarrow 100 \mathrm{~K}$.

4. The gas kinetic temperatures derived from the para$\mathrm{H}_{2} \mathrm{CO}(3-2)$ line ratios agree with the warm dust components measured at mid-infrared wavelength, indicating that the para- $\mathrm{H}_{2} \mathrm{CO}(3-2)$ ratios trace denser and warmer gas than $\mathrm{NH}_{3}(2,2) /(1,1)$ and $\mathrm{CH}_{3} \mathrm{CCH}(6-5)$. The cold dust components measured in the far infrared are consistent with those deduced from $\mathrm{NH}_{3}(2,2) /(1,1)$ and $\mathrm{CH}_{3} \mathrm{CCH}(6-5)$. Based on observations of dust emission at MIR and FIR wavelengths and molecular gas with para$\mathrm{H}_{2} \mathrm{CO}(3-2), \mathrm{NH}_{3}(2,2) /(1,1)$, and $\mathrm{CH}_{3} \mathrm{CCH}(6-5)$, dust and gas temperatures appear to be generally equivalent in the dense gas $\left(n\left(\mathrm{H}_{2}\right) \gtrsim 10^{4} \mathrm{~cm}^{-3}\right)$ of the OMC-1 region, but with the MIR continuum and para- $\mathrm{H}_{2} \mathrm{CO}$ likely sampling gas more closely associated with massive star formation.

5. The non-thermal velocity dispersions of para- $\mathrm{H}_{2} \mathrm{CO}$ are positively correlated with the gas kinetic temperatures at density $10^{5} \mathrm{~cm}^{-3}$ in regions of strong non-thermal motion (Mach number $\gtrsim 2.5$ ) in OMC-1, which implies that the higher temperature traced by para- $\mathrm{H}_{2} \mathrm{CO}$ is related to turbulence on a scale of $\sim 0.06 \mathrm{pc}$. Turbulent heating seems to be widespread in massive star formation regions on a $\sim 0.1-2$ pc scale.

6. Combining the temperature measurements with para$\mathrm{H}_{2} \mathrm{CO}(3-2)$ and $\mathrm{NH}_{3}(2,2) /(1,1)$ line ratios, we find direct evidence for the dense gas along the northern part of the OMC-1 $10 \mathrm{~km} \mathrm{~s}^{-1}$ filament being heated by radiation from the central Orion nebula.

Acknowledgements. The authors are grateful for the valuable comments of the referee. We thank the staff of the APEX telescope for their assistance in observations. We also thank Arnaud Belloche and Cosmos Yeh for their help of observations and data reduction. The authors are thankful for the helpful comments of Jens Kauffmann. This work acknowledges support by The Program of the Light in China's Western Region (LCRW) under grant XBBS201424 and The National Natural Science Foundation of China under grant 11433008 and 11373062. This work was partially carried out within the Collaborative Research Council 956, subproject A6, funded by the Deutsche Forschungsgemeinschaft (DFG). C.H. acknowledges support by a Chinese Academy of Sciences President's International Fellowship Initiative for visiting scientists (2017VMA0005). Y.G. acknowledges support by The National Natural Science Foundation of China under 
grant 11127903 and The National Key Research and Development program under grant 2017YFA0402702. This research has used NASA's Astrophysical Data System (ADS).

\section{References}

Ao, Y., Henkel, C., Menten, K. M., et al. 2013, A\&A, 550, A135 Baan, W. A., Güsten, R., \& Haschick, A. D. 1986, ApJ, 305, 830 Baan, W. A., Henkel, C., Schilke, P., et al. 1990, ApJ, 353, 132 Baan, W. A., Haschick, A. D., \& Uglesich, R. 1993, ApJ, 415, 140 Bally, J., \& Zinnecker, H. 2005, AJ, 129, 2281

Bally, J., Langer, W. D., Stark, A. A., \& Wilson, R. W. 1987, ApJ, 312, L45 Bally, J., Ginsburg, A., Arce, H., et al. 2017, ApJ, 837, 60

Bastien, P., Batrla, W., Henkel, C., et al. 1985, A\&A, 146, 86 Batrla, W., \& Wilson, T. L. 2003, A\&A, 408, 231

Batrla, W., Wilson, T. L., Ruf, K., \& Bastien, P. 1983, A\&A, 128, 279 Battersby, C., Bally, J., Dunham, M., et al. 2014, ApJ, 786, 116 Bendo, G. J., Boselli, A., Dariush, A., et al. 2012, MNRAS, 419, 1833 Benson, P. J., \& Myers, P. C. 1983, ApJ, 270, 589

Bergin, E. A., Goldsmith, P. F., Snell, R. L., et al. 1994, ApJ, 431, 674 Bergin, E. A., Snell, R. L., \& Goldsmith, P. F. 1996, ApJ, 460, 343 Bergman, P., Parise, B., Liseau, R., \& Larsson, B. 2011, A\&A, 527, A39 Berné, O., Marcelino, N., \& Cernicharo, J. 2014, ApJ, 795, 13 Bieging, J. H., Wilson, T. L., \& Downes, D. 1982, A\&AS, 49, 607 Buckle, J. V., Davis, C. J., Francesco, J. D., et al. 2012, MNRAS, 422, 521 Caselli, P., Hasegawa, T. I., \& Herbst, E. 1993, ApJ, 408, 548 Chira, R. A., Beuther, H., Linz, H., et al. 2013, A\&A, 552, A40 Churchwell, E., \& Hollis, J. M. 1983, ApJ, 272, 591 Cohen, R. J., \& Few, R. W. 1981, MNRAS, 194, 71 Cohen, R. J., Matthews, N., Few, R. W., et al. 1983, MNRAS, 203, 1123 Danby, G., Flower, D. R., Valiron, P., et al. 1988, MNRAS, 235, 229 Dewangan, L. K., Ojha, D. K., Luna, A., et al. 2016, ApJ, 819, 66 Downes, D., Wilson, T. L., Bieging, J., \& Wink, J. 1980, A\&AS, 40, 379 Downes, D., Genzel, R., Becklin, E. E., et al. 1981, ApJ, 244, 869 Dunham, M. K., Rosolowsky, E., Evans, II, N. J., et al. 2010, ApJ, 717, 1157 Friesen, R., Pineda, J. E., Rosolowsky, E., et al. 2017, ApJ, 843, 63 Gerner, T., Beuther, H., Semenov, D., et al. 2014, A\&A, 563, A97 Giannetti, A., Brand, J., Sánchez-Monge, Á., et al. 2013, A\&A, 556, A16 Giannetti, A., Leurini, S., Wyrowski, F., et al. 2017, A\&A, 603, A33 Ginsburg, A., Darling, J., Battersby, C., et al. 2011, ApJ, 736, 149 Ginsburg, A., Bally, J., Battersby, C., et al. 2015, A\&A, 573, A106 Ginsburg, A., Henkel, C., Ao, Y., et al. 2016, A\&A, 586, A50 Ginsburg, A., Goddi, C., Kruijssen, J. D., et al. 2017, ApJ, 842, 92 Goicoechea, J. R., Teyssier, D., Etxaluze, M., et al. 2015, ApJ, 812, 75 Goldsmith, P. F. 2001, ApJ, 557, 736

Goldsmith, P. F., Bergin, E. A., \& Lis, D. C. 1997, ApJ, 491, 615 Gong, Y., Henkel, C., Spezzano, S., et al. 2015a, A\&A, 574, A56 Gong, Y., Henkel, C., Thorwirth, S., et al. 2015b, A\&A, 581, A48 Guo, W. H., Esimbek, J., Tang, X. D., et al. 2016, Ap\&SS, 361, 264 Güsten, R., Walmsley, C. M., Ungerechts, H., et al. 1985, A\&A, 142, 381 Guzmán, A. E., Sanhueza, P., Contreras, Y., et al. 2015, ApJ, 815, 130 Hacar, A., Alves, J., Tafalla, M., \& Goicoechea, J. R. 2017, A\&A, 602, L2 He, Y. X., Zhou, J. J., Esimbek, J., et al. 2016, MNRAS, 461, 2288 Helou, G. 1986, ApJ, 311, L33

Henkel, C., Walmsley, C. M., \& Wilson, T. L. 1980, A\&A, 82, 41 Henkel, C., Wilson, T. L., Walmsley, C. M., \& Pauls, T. 1983, A\&A, 127, 388 Henkel, C., Wouterloot, J. G. A., \& Bally, J. 1986, A\&A, 155, 193 Henkel, C., Wilson, T. L., \& Mauersberger, R. 1987, A\&A, 182, 137 Henkel, C., Baan, W. A., \& Mauersberger, R. 1991, A\&ARv, 3, 47 Ho, P. T. P., \& Townes, C. H. 1983, ARA\&A, 21, 239

Hurt, R., Barsony, M., \& Wootten, A. 1996, ApJ, 456, 686 Immer, K., Galván-Madrid, R., König, C., et al. 2014, A\&A, 572, A63 Immer, K., Kauffmann, J., Pillai, T., et al. 2016, A\&A, 595, A94 Jijina, J., Myers, P. C., \& Adams, F. C. 1999, ApJS, 125, 161 Johnston, K. G., Beuther, H., Linz, H., et al. 2014, A\&A, 568, A56 Johnstone, D., \& Bally, J. 1999, ApJ, 510, L49

Johnstone, D., Boonman, A. M. S., \& van Dishoeck, E. F. 2003, A\&A, 412, 157 Kainulainen, J., Stutz, A. M., Stanke, T., et al. 2017, A\&A, 600, A141 Kauffmann, J., Goldsmith, P. F., Melnick, G., et al. 2017, A\&A, 605, L5 König, C., Urquhart, J. S., Csengeri, T., et al. 2017, A\&A, 599, A139 Kounkel, M., Hartmann, L., Loinard, L., et al. 2017, ApJ, 834, 142 Kutner, M. L., Evans, N. J., II, \& Tucker, K. D. 1976, ApJ, 209, 452 Lada, C. J., Bergin, E. A., Alves, J. F., \& Huard, T. L. 2003, ApJ, 586, 286 Leurini, S., Parise, B., Schilke, P., Pety, J., \& Rolffs, R. 2010, A\&A, 511, A82 Lin, Y. X, Liu, H. B., Li, D., et al. 2016, ApJ, 828, 32 Lindberg, J. E., \& Jørgensen, J. K. 2012, A\&A, 548, A24
Lindberg, J. E., Jørgensen, J. K., Watanabe, Y., et al. 2015, A\&A, 584, A28 Lis, D. C., Serabyn, E., Keene, J., et al. 1998, ApJ, 509, 299 Lombardi, M., Bouy, H., Alves, J., \& Lada, C. J. 2014, A\&A, 566, A45 Lu, X., Zhang, Q., Liu, H. B., Wang, J., \& Gu, Q. 2014, ApJ, 790, 84 Lu, X., Zhang, Q., Kauffmann, J., et al. 2017, ApJ, 839, 1 Mangum, J. G., \& Wootten, A. 1993, ApJS, 89, 123 Mangum, J. G., Wootten, A., Wadiak, E. J., \& Loren, R. B. 1990, ApJ, 348, 542 Mangum, J. G., Wootten, A., \& Plambeck, R. L. 1993, ApJ, 409, 282 Mangum, J. G., Wootten, A., \& Barsony, M. 1999, ApJ, 526, 845 Mangum, J. G., Darling, J., Menten, K. M., \& Henkel, C. 2008, ApJ, 673, 832 Mangum, J. G., Darling, J., Henkel, C., et al. 2013a, ApJ, 779, 33 Mangum, J. G., Darling, J., Henkel, C., et al. 2013b, ApJ, 766, 108 Martín-Pintado, J., Rodríguez-Franco, A., \& Bachiller, R. 1990, ApJ, 357, 49 Mauersberger, R., Henkel, C., Wilson, T. L., et al. 1986, A\&A, 162, 199 Mauersberger, R., Henkel, C., \& Wilson, T. L. 1987, A\&A, 173, 352 Megeath, S. T., Gutermuth, R., Muzerolle, J., et al. 2012, AJ, 144, 192 Melnick, G. J., Tolls, V., Snell, R. L., et al. 2011, ApJ, 727, 13 Menten, K. M., Reid, M. J., Forbrich, J., \& Brunthaler, A. 2007, A\&A, 474, 515 Merello, M., Evans, N. J. II., Shirley, Y. L., et al. 2015, ApJS, 218, 1 Mitchell, G. F., Johnstone, D., Moriarty-Schieven, G., et al. 2001, ApJ, 556, 215 Molinari, S., Brand, J., Cesaroni, R., \& Palla, F. 1996, A\&A, 308, 573 Mookerjea, B., Ghosh, S. K., Rengarajan, T. N., et al. 2000, AJ, 120, 1954 Mühle, S., Seaquist, E. R., \& Henkel, C. 2007, ApJ, 671, 1579 Murata, Y., Kawabe, R., Ishiguro, M., et al. 1990, ApJ, 359, 125 Nagy, Z., van der Tak, F. F. S., Fuller, G. A., et al. 2012, A\&A, 542, A6 Nishimura, A., Tokuda, K., Kimura, K., et al. 2015, ApJS, 216, 18

O’Dell, C. R., Muench, A., Smith, N., \& Zapata, L. 2008, Handbook of Star Forming Regions, Vol. I: The Northern Sky ASP Monograph Publications, Vol. 4, ed. B. Reipurth (San Francisco: ASP), 544

Ott, J., Weiss, A., Staveley-Smith, L., et al. 2014, ApJ, 785, 16

Pan, L., \& Padoan, P. 2009, ApJ, 692, 594

Peng, T. C., Wyrowski, F., Zapata, L. A., et al. 2012, A\&A, 538, A12

Qin, S. L., Zhao, J. H., Moran, J. M., et al. 2008, ApJ, 677, 353

Rodríguez-Franco, A., Martín-Pintado, J., Gómez-Gínzalez, J., \& Planesas, P. 1992, A\&A, 264, 592

Salgado, F., Berné, O., Adams, J. D., et al. 2016, ApJ, 830, 118

Salji, C. J., Richer, J. S., Buckle, J. V., et al. 2015, MNRAS, 449, 1782

Schilke, P., Walmsley, C. M., Pineau Des Forets, G., et al. 1992, A\&A, 256, 595

Schnee, S., Rosolowsky, E., Foster, J., Enoch, M., \& Sargent, A. 2009, ApJ, 691, 1754

Shimajiri, Y., Kawabe, R., Takakuwa, S., et al. 2011, PASJ, 63, 105

Shimajiri, Y., Kitamura, Y., Saito, M., et al. 2014, A\&A, 564, A68

Shirley, Y. L. 2015, PASP, 127, 299

Stutz, A. M., \& Kainulainen, J. 2015, A\&A, 577, L6

Tang, X. D., Esimbek, J., Zhou, J. J., et al. 2013, A\&A, 551, A28

Tang, X. D., Henkel, C., Menten, K. M., et al. 2017a, A\&A, 598, A30 (Paper I)

Tang, X. D., Henkel, C., Chen, C.-H. R., et al. 2017b, A\&A, 600, A16 (Paper II)

Tang, X. D., Henkel, C., Wyrowski, F., et al. 2018, A\&A, in press, DOI: $10.1051 / 0004-6361 / 201732168$

Tatematsu, K., Umemoto, T., Kameya, O., et al. 1993, ApJ, 404, 643 Tatematsu, K., Umemoto, T., Heyer, M. H., et al. 1998, ApJS, 118, 517 Tatematsu, K., Kandori, R., Umemoto, T., \& Sekimoto, Y. 2008, PASJ, 60, 407 Thaddeus, P., Wilson, R. W., Kutner, M., et al. 1971, ApJ, 168, 59 Treviño-Morales, S. P., Pilleri, P., Fuente, A., et al. 2014, A\&A, 569, A19 Ungerechts, H., Bergin, E. A., Goldsmith, P. F., et al. 1997, ApJ, 482, 245 Urquhart, J. S., Morgan, L. K., Figura, C. C., et al. 2011, MNRAS, 418, 1689 Urquhart, J. S., Figura, C. C., Moore, T. J. T., et al. 2015, MNRAS, 452, 4029 Vaillancourt, J. E. 2002, ApJS, 142, 53

van der Tak, F. F. S., Black, J. H., Schöier, F. L., et al. 2007, A\&A, 468, 627 van der Wiel, M. H. D., van der Tak, F. F. S., Ossenkopf, V., et al. 2009, A\&A, 498, 161

Walmsley, C. M., \& Ungerechts, H. 1983, A\&A, 122, 164

Wang, Y., Beuther, H., Zhang, Q., et al. 2012, ApJ, 754, 87

Watanabe, T., \& Mitchell, G. 2008, AJ, 136, 1947

Weiß, A., Neininger, N., Henkel, C., et al. 2001, ApJ, 554, L143

Wienen, M., Wyrowski, F., Schuller, F., et al. 2012, A\&A, 544, A146 Wiesenfeld, L., \& Faure, A. 2013, MNRAS, 432, 2573

Wilson, B. A., Dame, T. M., Masheder, M. R. W., et al. 2005, A\&A, 430, 523 Wiseman, J. J., \& Ho, P. T. P. 1996, Nature, 382, 139 Wiseman, J. J., \& Ho, P. T. P. 1998, ApJ, 502, 676

Wouterloot, J. G. A., Walmsley, C. M., \& Henkel, C. 1988, A\&A, 203, 367 Wu, Y., Zhang, Q., Yu, W., et al. 2006, A\&A, 450, 607 Yu, N., \& Xu, J. 2016, ApJ, 833, 248

Zapata, L. A., Rodríguez, L. F., Kurtz, S. E., et al. 2004, ApJ, 610, L121 Zapata, L. A., Schmid-Burgk, J., \& Menten, K. M. 2011, A\&A, 529, A24 Zhang, C. P., Esimbek, J., Zhou, J. J., et al. 2012, Ap\&SS, 337, 283 Zylka, R., Güsten, R., Henkel, C., \& Batrla, W. 1992, A\&AS, 96, 525 


\section{Appendix A: $\mathrm{H}_{2} \mathrm{CO}$ velocity channel maps}

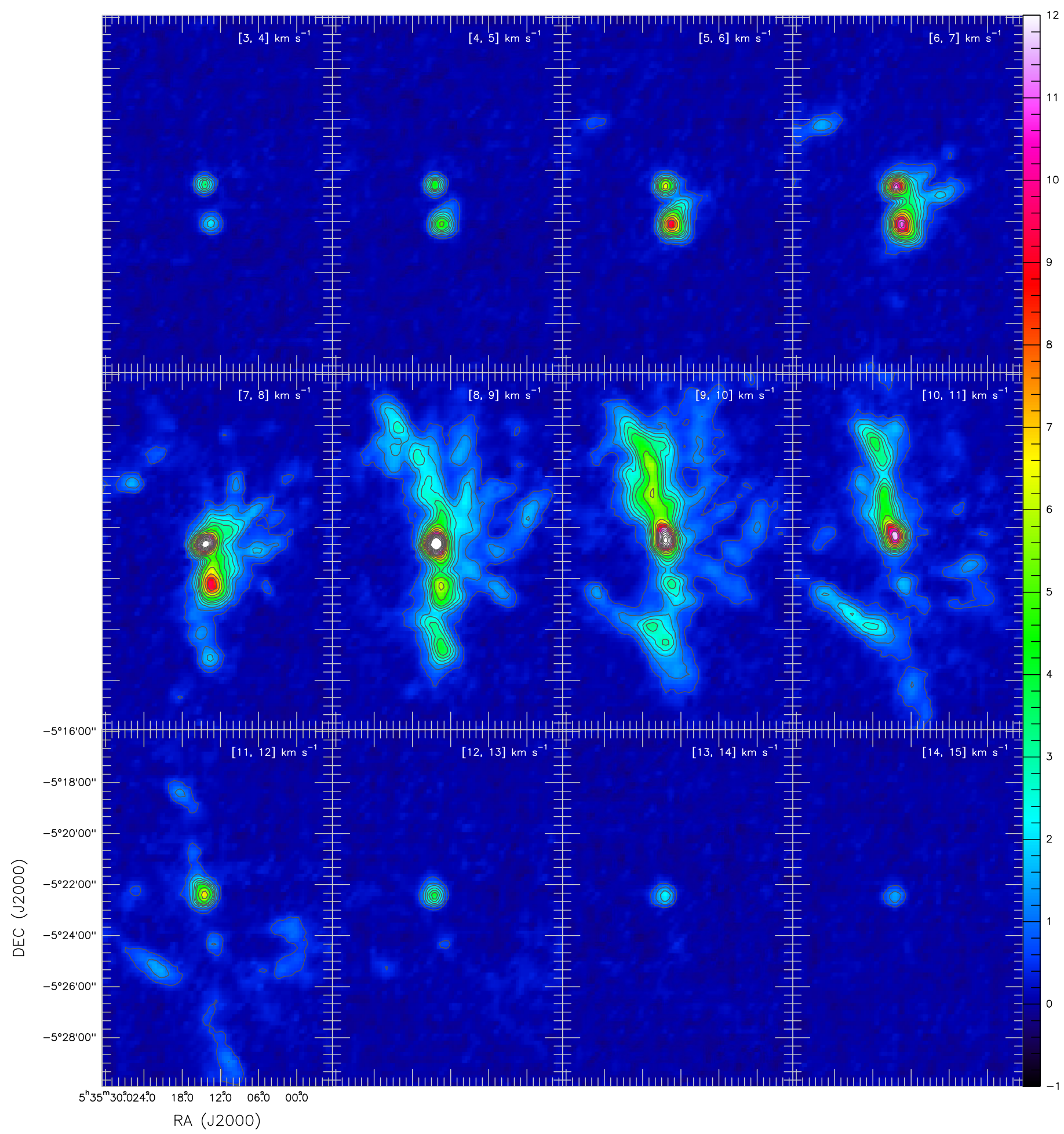

Fig. A.1. Channel maps of the para- $\mathrm{H}_{2} \mathrm{CO}_{303}-2_{02}$ transition. The contours are running from 0.5 to $2.9 \mathrm{~K}$ in steps of $0.6 \mathrm{~K}$ and from 3.5 to $14.3 \mathrm{~K}$ in steps of $1.2 \mathrm{~K}\left(T_{\mathrm{A}}^{*}\right.$ scale; color bar in units of $\left.\mathrm{K}\right)$. 


\section{Appendix B: Comparison of $\mathrm{H}_{2} \mathrm{CO}, \mathrm{NH}_{3}$, and dust distributions}

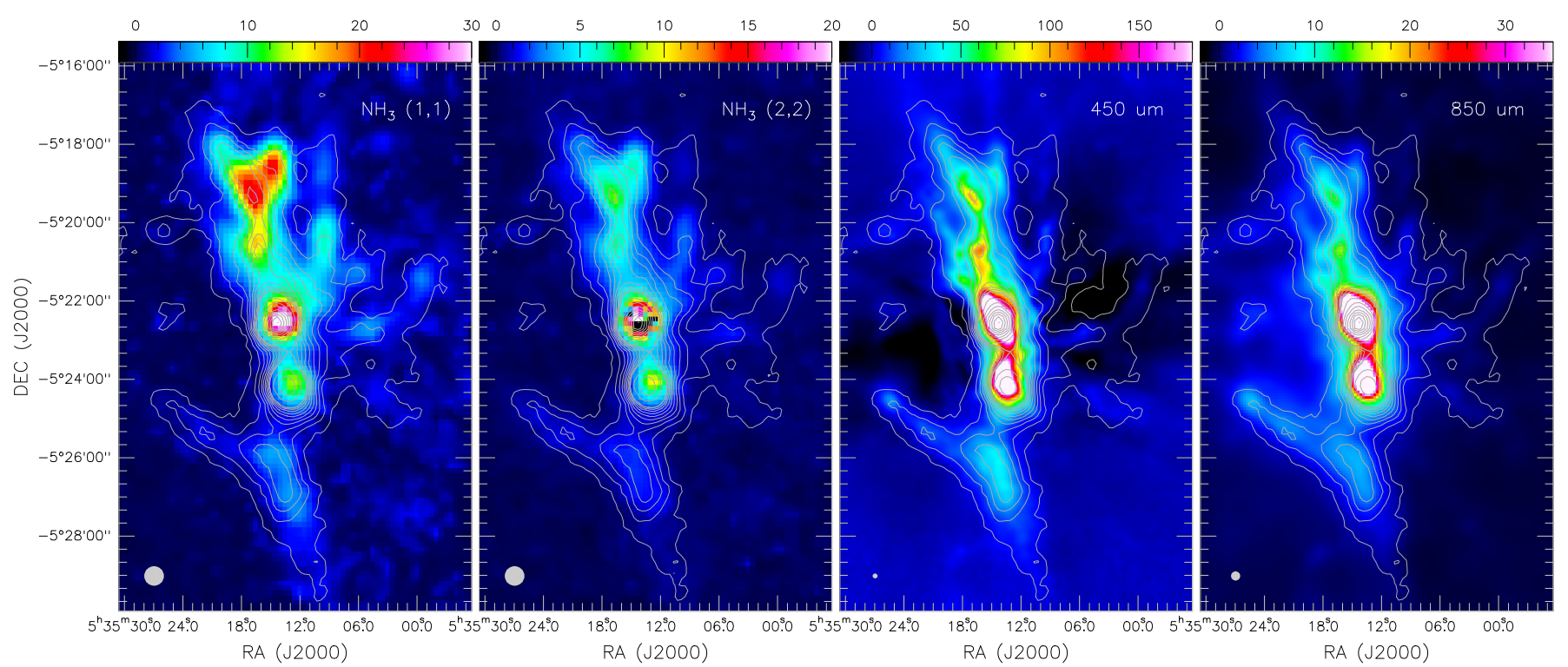

Fig. B.1. Para- $\mathrm{H}_{2} \mathrm{CO}\left(3_{03}-2_{02}\right)$ with integrated intensity contours (same as in Fig. 2) overlaid on $\mathrm{NH}_{3}(1,1)$, and $(2,2)$ integrated intensity observed with the GBT (beam size 30"; left panels; Friesen et al. 2017), and JCMT/SCUBA 450 and $850 \mu \mathrm{m}$ dust emission (beam sizes 7.5" and 14"; right panels; Johnston \& Bally 1999) images of the OMC-1. The beam of each image is shown in the lower left corner.

\section{Appendix C: Comparison of para- $\mathrm{H}_{2} \mathrm{CO}_{22}-2_{21}$ and $3_{21}-2_{20}$ line profiles}
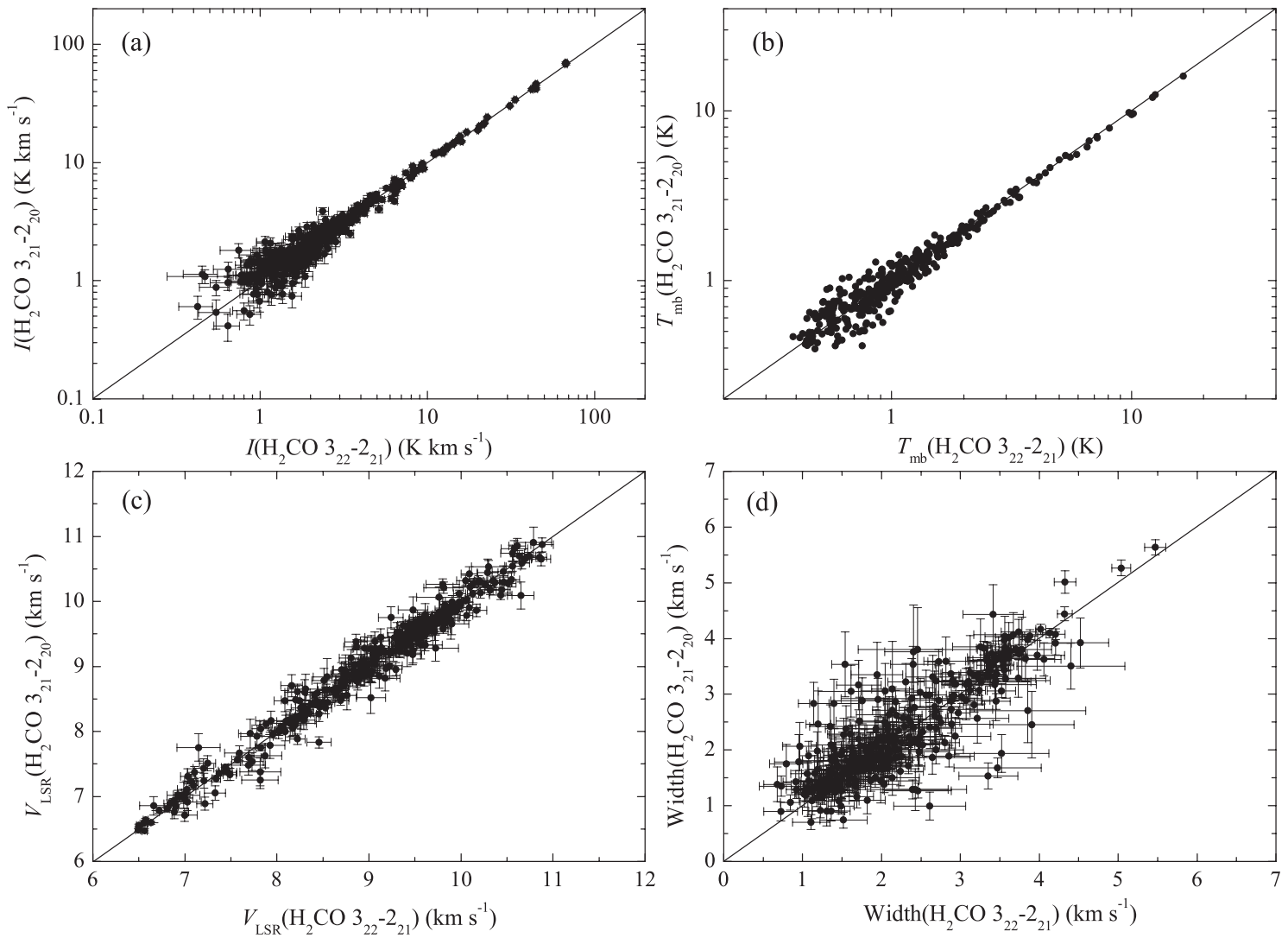

Fig. C.1. Comparisons of integrated intensities $(a)$, line brightness temperatures $(b)$, velocities $(c)$, and linewidths $(d)$ of para- $\mathrm{H}_{2} \mathrm{CO} 3_{22}-2_{21}$ and $3_{21}-2_{20}$ lines. The straight lines indicate $Y=X$. 
Appendix D: Comparison of gas kinetic temperatures derived from para- $\mathrm{H}_{2} \mathrm{CO}$ and $\mathrm{NH}_{3}$
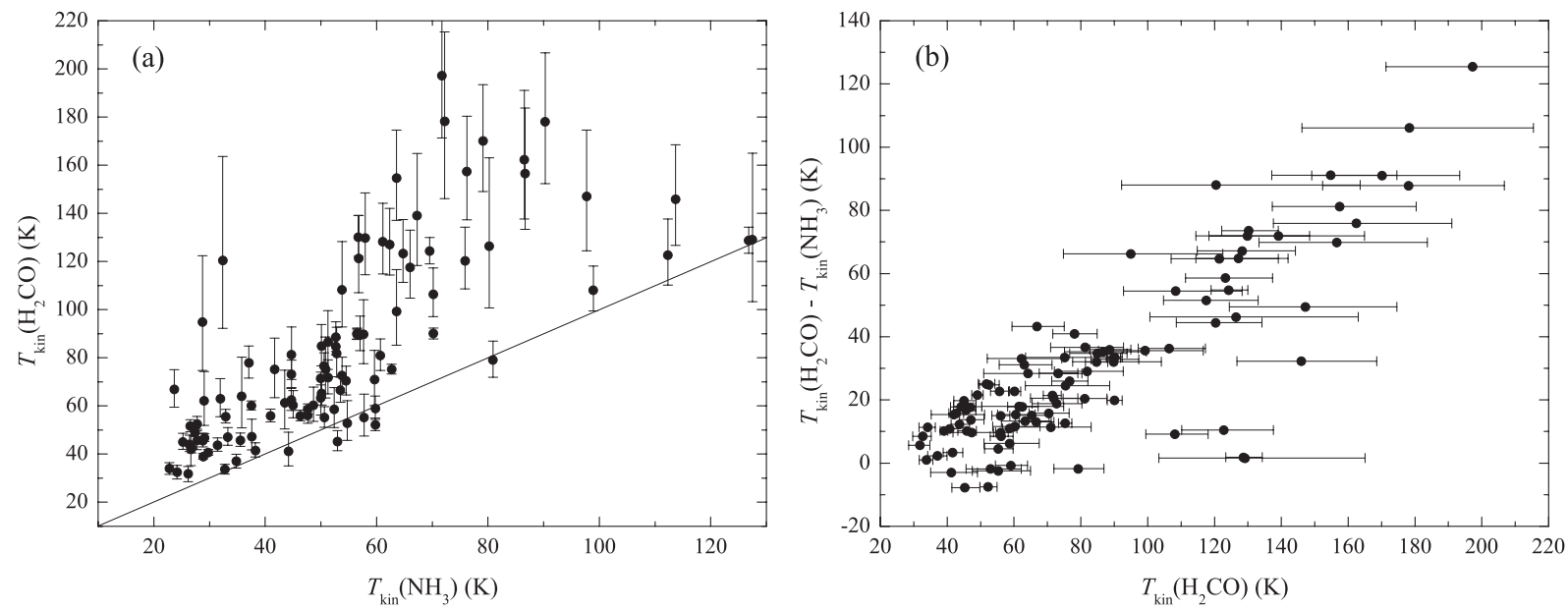

Fig. D.1. Comparison of gas kinetic temperatures $(a)$ and relative gas kinetic temperature difference $(b)$ derived from para- $\mathrm{H}_{2} \mathrm{CO}(3-2)$ and $\mathrm{NH}_{3}(2,2) /(1,1)$ line ratios. The straight line indicates same temperature. 